\title{
A new bioavailable fenretinide formulation with antiproliferative, antimetabolic, and cytotoxic effects on solid tumors
}

\author{
Isabella Orienti ${ }^{1}$, Federica Francescangelii, Maria Laura De Angelis ${ }^{2}$, Katia Fecchi ${ }^{3}$, Lucilla Bongiorno-Borbone ${ }^{4}$, \\ Michele Signore $\mathbb{0}^{5}$, Angelo Peschiaroli ${ }^{4}$, Alessandra Boe ${ }^{6}$, Alessandro Bruselles ${ }^{2}$, Angelita Costantino ${ }^{2,7}$, \\ Adriana Eramo², Valentina Salvati ${ }^{2}$, Giovanni Sette ${ }^{2}$, Paola Contavalli², Lello Zolla ${ }^{8}$, Toshihiko Oki ${ }^{9,10}$, Toshio Kitamura ${ }^{9,10,}$ \\ Massimo Spada ${ }^{11}$, Alessandro Giuliani ${ }^{12}$, Marta Baiocchi ${ }^{2}$, Filippo La Torre ${ }^{13}$, Gerry Melino $\mathbb{E}^{4}$, Marco Tartaglia ${ }^{14}$, \\ Ruggero De Maria ${ }^{15}$ and Ann Zeuner $\mathbb{1}^{2}$
}

\begin{abstract}
Fenretinide is a synthetic retinoid characterized by anticancer activity in preclinical models and favorable toxicological profile, but also by a low bioavailability that hindered its clinical efficacy in former clinical trials. We developed a new formulation of fenretinide complexed with 2-hydroxypropyl-beta-cyclodextrin (nanofenretinide) characterized by an increased bioavailability and therapeutic efficacy. Nanofenretinide was active in cell lines derived from multiple solid tumors, in primary spheroid cultures and in xenografts of lung and colorectal cancer, where it inhibited tumor growth independently from the mutational status of tumor cells. A global profiling of pathways activated by nanofenretinide was performed by reverse-phase proteomic arrays and lipid analysis, revealing widespread repression of the mTOR pathway, activation of apoptotic, autophagic and DNA damage signals and massive production of dihydroceramide, a bioactive lipid with pleiotropic effects on several biological processes. In cells that survived nanofenretinide treatment there was a decrease of factors involved in cell cycle progression and an increase in the levels of p16 and phosphorylated p38 MAPK with consequent block in G0 and early G1. The capacity of nanofenretinide to induce cancer cell death and quiescence, together with its elevated bioavailability and broad antitumor activity indicate its potential use in cancer treatment and chemoprevention.
\end{abstract}

Over the last decades, the use of targeted agents and immunotherapeutics has increasingly flanked classic cytotoxics, leading to a significant survival improvement

\footnotetext{
Correspondence: Ruggero De Maria (ruggerodemaria@gmail.com) or Ann Zeuner (a.zeuner@gmail.com)

'Department of Pharmacy and Biotechnology, University of Bologna via San Donato 19/2, 40127 Bologna, Italy

${ }^{2}$ Department of Oncology and Molecular Medicine, Istituto Superiore di Sanità, Viale Regina Elena 299, 00161 Rome, Italy

Full list of author information is available at the end of the article.

These authors contributed equally: Isabella Orienti, Federica Francescangeli, Maria Laura De Angelis

These authors jointly supervised this work: Katia Fecchi, Lucilla BongiornoBorbone

Edited by M. Agostini
}

for selected subsets of cancer patients. Nevertheless, new therapies are often associated to high economic burden, toxic side effects and absence of predictive biomarkers of efficacy. Therapy failure in cancer patients is tightly linked to the presence of tumor-initiating cells responsible for drug resistance and cancer relapse ${ }^{1,2}$. Therefore, effective therapeutic strategies should ideally be able not only to kill cancer cells but also to prevent the reactivation of remaining tumorigenic cells. Fenretinide (N-4-hydroxyphenyl-retinamide) is a synthetic derivative of all-trans retinoic acid characterized by high cytotoxic efficacy against cancer cells in vitro and previously investigated as a potential anticancer and chemopreventive $\operatorname{drug}^{3-11}$.

\section{(c) The Author(s) 2019}

(c) (i) Open Access This article is licensed under a Creative Commons Attribution 4.0 International License, which permits use, sharing, adaptation, distribution and reproduction cc) in any medium or format, as long as you give appropriate credit to the original author(s) and the source, provide a link to the Creative Commons license, and indicate if changes were made. The images or other third party material in this article are included in the article's Creative Commons license, unless indicated otherwise in a credit line to the material. If material is not included in the article's Creative Commons license and your intended use is not permitted by statutory regulation or exceeds the permitted use, you will need to obtain permission directly from the copyright holder. To view a copy of this license, visit http://creativecommons.org/licenses/by/4.0/. 
Previous clinical phase I-III evaluations of fenretinide have shown minimal systemic toxicity and good tolerability $^{7,12,13}$, fostering additional studies aimed at exploiting the selective anticancer effect of this compound. However, clinical trials aimed at evaluating the activity of fenretinide in cancer patients yielded frustrating results as therapeutic plasma levels could not be attained due to the poor aqueous solubility and consequent low bioavailability of the drug ${ }^{3,8,13}$. In fact plasma concentrations of fenretinide remained below the minimum threshold for the onset of the antitumor activity also after multiple and protracted administrations $\mathrm{s}^{3,13}$. Formulations aimed at increasing fenretinide bioavailability were previously prepared by drug incorporation into lipid matrices or oil-in-water emulsions. They showed an improved performance compared to previous studies but faced some problems related to the increase of the administration dose and the correspondent increase in plasma concentration ${ }^{14-17}$. We have prepared a new fenretinide formulation characterized by improved aqueous solubility through drug salification and complexation with 2-hydroxypropyl $\beta$-cyclodextrin, a solubilizing excipient endowed with favorable biodistribution and reduced toxicity ${ }^{18}$. The new fenretinide formulation (referred thereafter as nanofenretinide, NanoFEN) showed high anticancer efficacy in vitro and in vivo against cell lines from multiple tumors, primary spheroid cultures of lung and colon cancer cells and tumor xenografts in the absence of macroscopic toxic effects. A global analysis of NanoFEN-activated events revealed a widespread inhibition of the mTOR pathway, cell death with mixed autophagic/apoptotic features and cell cycle block with induction of quiescence. Such events occurred in parallel with a massive accumulation of bioactive dihydroceramide lipids, pleiotropic inhibitors of cell cycle and metabolism. Altogether, these results indicate that NanoFEN activates a multifactorial program in cancer cells composed by signals of apoptosis, autophagy and proliferative/metabolic inhibition, resulting in a widespread and durable antitumor effect. Although additional studies will be required to establish the efficacy and absence of toxicity of NanoFEN in human subjects, our results indicate this compound as a candidate for future clinical studies.

\section{Results}

Generation of a new bioavailable fenretinide formulation

The extremely poor aqueous solubility of fenretinide traditionally represents a strong drawback limiting the bioavailability of this molecule and its use in anticancer therapy. A possible solution for increasing fenretinide bioavailability is its complexation with cyclodextrins, in order to improve the drug aqueous solubility and provide chemical stability. Compared to other solubilizing excipients marketed in pharmaceutical products, cyclodextrins are endowed with favorable biodistribution and reduced toxicity also after repeated administrations ${ }^{18}$. However, the very low solubility of fenretinide in water prevents its spontaneous inclusion into the hydrophobic cavity of the cyclodextrin, compromising the effectiveness of the complexation process. We overcame this obstacle by rising the aqueous solubility of fenretinide through an unprecedented procedure of fenretinide salification that allowed drug complexation to take place in the presence of hydroxypropyl $\beta$-cyclodextrin (Fig. 1a). The formation of the fenretinide-cyclodextrin complex is demonstrated by the chemical shift changes in the nuclear magnetic resonance $\left({ }^{1} \mathrm{H}\right.$ NMR) spectrum corresponding to protons $\mathrm{H} 8, \mathrm{H} 3, \mathrm{H} 5, \mathrm{H} 2, \mathrm{H} 6$ of the complexed fenretinide (Fig. 1b, right panel) with respect to the fenretinide salt (Fig. 1b, left panel). Figure 1c reports the aqueous solubility of NanoFEN, showing it is $>1000$ times higher than pure fenretinide (PureFEN). Importantly, in order to provide an effective antitumor activity, the NanoFEN complex should be stable in aqueous body fluids and ideally release its active molecule only after accumulation in the tumor site, behaving as an hydrophobic absorbing phase and favouring accumulation by the enhanced permeability and retention effect (EPR effect) ${ }^{19}$. Figure 1d shows that the release of fenretinide from the complex takes place only in the presence of an adsorbing hydrophobic phase while it is suppressed in a plain aqueous environment, thus supporting a productive use of this formulation in the antitumor therapy. Pharmacokinetics and bioavailability of NanoFEN in mice were assessed by comparing intravenous administration of NanoFEN versus fenretinide contained in the oral gelatin capsules provided by the National Cancer Institute and previously used in the majority of clinical trials, which contain fenretinide dispersed in corn oil and polysorbate-80 (referred thereafter as NCI fenretinide). We compared intravenous administration of NanoFEN versus oral administration of NCI fenretinide (Fig. 1e, left), oral administration of NanoFEN versus oral administration of NCI fenretinide (Fig. 1e, center) and intravenous administration of NanoFEN versus intravenous administration of PureFEN (Fig. 1e, right). The latter was dissolved in $10 \%$ ethanol and saline to obtain drug solubilization, although this procedure could not be applicable to human use due to the presence of fenretinide precipitates. In all three cases, NanoFEN provided significantly higher drug exposure, being Area Under the Curve (AUC) values obtained after NanoFEN 
A

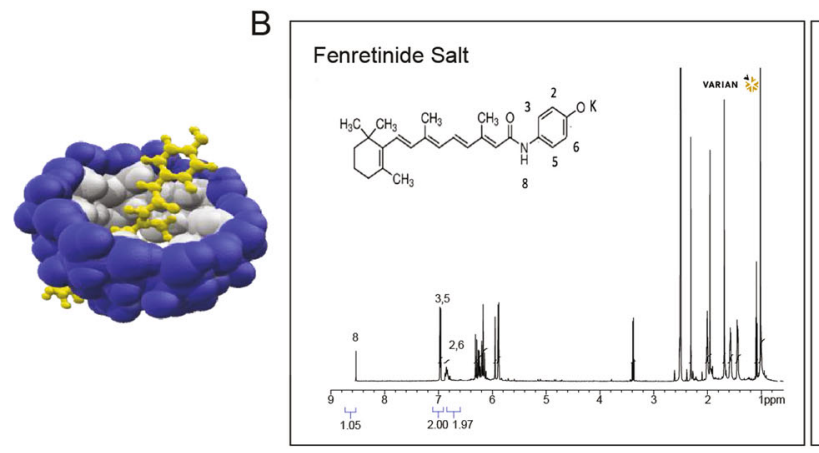

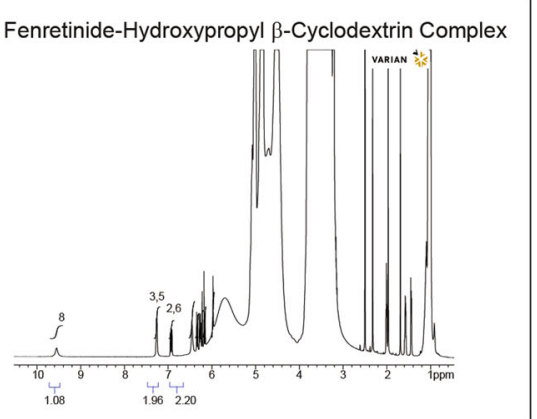

C

\begin{tabular}{|l|c|}
\hline Solubility in $\mathrm{H}_{2} \mathrm{O}$ & $\boldsymbol{\mu g} / \mathbf{m l}$ \\
\hline PureFEN & $1,71 \pm 0,08$ \\
\hline NanoFEN & $2410 \pm 0,86$ \\
\hline
\end{tabular}
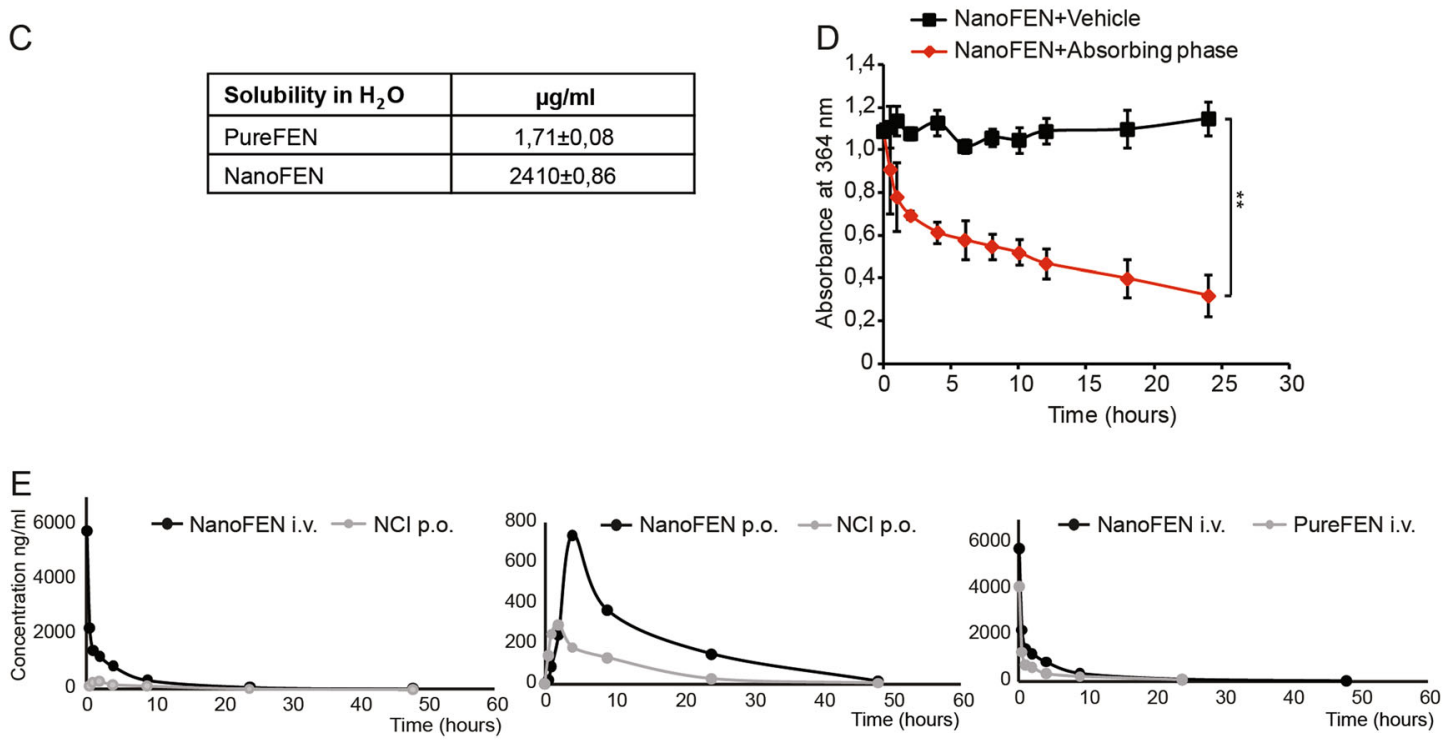

\begin{tabular}{|l|c|c|l|}
\hline & $\begin{array}{c}\text { NanoFEN } \\
\text { i.v. }\end{array}$ & $\begin{array}{c}\text { NCl } \\
\text { p.o. }\end{array}$ & \multicolumn{1}{|}{} \\
\hline Cmax & 6932,6 & 298,13 & ng/mI \\
\hline AUC & 13656,8 & 3209,7 & hr* $\mathbf{n g} / \mathbf{m l}$ \\
\hline
\end{tabular}

\begin{tabular}{|l|c|c|l|}
\hline & $\begin{array}{c}\text { NanoFEN } \\
\text { p.o. }\end{array}$ & $\begin{array}{c}\text { NCI } \\
\text { p.o. }\end{array}$ & \multicolumn{1}{|l|}{} \\
\hline Cmax & 730,06 & 298,13 & ng/ml \\
\hline AUC & 9382,5 & 3209,7 & hr $^{*} \mathrm{ng} / \mathrm{ml}$ \\
\hline
\end{tabular}

\begin{tabular}{|l|c|c|l|} 
& $\begin{array}{c}\text { NanoFEN } \\
\text { i.v. }\end{array}$ & $\begin{array}{c}\text { PureFEN } \\
\text { i.v }\end{array}$ & \multicolumn{1}{|c|}{} \\
\hline Cmax & 6932,6 & 4134,77 & $\mathbf{n g} / \mathbf{m l}$ \\
\hline AUC & 13656,8 & 6574,35 & hr* $^{*} \mathbf{g} / \mathrm{ml}$ \\
\hline
\end{tabular}

Fig. 1 Generation and analysis of a new bioavailable fenretinide formulation. a Tridimensional representation of fenretinide (yellow) complexed with 2-hydroxypropyl- $\beta$-cyclodextrin (blue). $\mathbf{b}^{1} \mathrm{H}$ NMR analysis of fenretinide salt (left panel) and fenretinide complex with cyclodextrin (right panel). Chemical shift changes corresponding to protons $\mathrm{H} 8, \mathrm{H} 3, \mathrm{H} 5, \mathrm{H} 2, \mathrm{H} 6$ of the complexed fenretinide (right panel). c Solubility of PureFEN and NanoFEN in water at $25^{\circ} \mathrm{C}$. $\mathbf{d}$ Release of fenretinide from NanoFEN in a plain aqueous environment (black squares, NanoFEN+Vehicle) or in the presence of an absorbing phase (red diamonds, NanoFEN+Absorbing phase). Values represent mean \pm SD of three independent experiments. ${ }^{* *} P<$ 0.01 from two-tailed t-test. e Plasma pharmacokinetic profiles of fenretinide after single administration of $5 \mathrm{mg} / \mathrm{kg}$ from different formulations: left panel, NanoFEN i.v. (black circles) compared to NCl p.o. (grey circles); central panel, NanoFEN p.o. (black circles) compared to NCl p.o. (grey circles); right panel, NanoFEN i.v. (black circles) compared to PureFEN i.v. (grey circles). Tables show the Cmax and AUC values of the relative graphs

administration always higher than those obtained with other systems either by oral or intravenous route (Fig. 1e and Table 1). Previous formulations of fenretinide used in clinical trials were characterized by a favorable toxicological profile, with side effects generally limited to diminished dark adaptation and dermatologic disorders even at high dosages ${ }^{7,12,13,20,21}$. In order to determine whether NanoFEN may produce macroscopic side effects in mice, we evaluated body weight, hepatic and hematologic parameters in mice treated intravenously with NanoFEN. Body weight, alanine transaminase (ALT), aspartate transaminase (AST), bilirubin and hematologic values at the end of the treatment were comparable to controls, providing preliminary evidences on the absence of systemic toxicity, hepatic damage and myelosuppression (Supplementary Fig. 1a-c). These results show that the new physico-chemical properties of NanoFEN provide a substantial increase in fenretinide plasma levels without 
Table 1 Main pharmacokinetic parameters of Fenretinide in CD1 female mice after administration of Nanofenretinide by oral or intravenous route in comparison with the $\mathrm{NCl}$ capsules and pure Fenretinide administered at the same dose ( $5 \mathrm{mg} / \mathrm{Kg}$ fenretinide)

\begin{tabular}{|c|c|c|c|c|c|}
\hline Pk parameters & NanoFEN i.v. & PureFEN i.v. & NanoFEN p.o. & NCI p.o. & Units \\
\hline AUC 0-last & 13563.5 & 6420.32 & 9378.9 & 3201 & $h^{*} n g / m$ \\
\hline$A \cup C$ inf & 13656.8 & 6574.35 & 9382.5 & 3209.7 & $h^{*} \mathrm{ng} / \mathrm{m}$ \\
\hline $\mathrm{Ke}$ & 0.094 & 0.088 & 0.106 & 0.089 & $1 / \mathrm{h}$ \\
\hline $\mathrm{HL}$ & 7.41 & 7.87 & 6.54 & 7.79 & h \\
\hline Tmax & - & - & 4 & 2 & h \\
\hline $\mathrm{Vd}$ & 3.9 & 6.52 & - & - & $\mathrm{L} / \mathrm{kg}$ \\
\hline Cmax & 6932.6 & 4134.77 & 730.06 & 298.13 & $\mathrm{ng} / \mathrm{ml}$ \\
\hline $\mathrm{Cl}$ & 0.366 & 0.574 & - & - & $\mathrm{L} / \mathrm{hr} / \mathrm{kg}$ \\
\hline
\end{tabular}

macroscopic side effects, although in-depth toxicological analyses will be required to rule out potential organspecific toxicities.

NanoFEN-induced killing of cancer cells from solid tumors

Multicellular spheroid cultures derived from primary tumors have been shown to constitute a reliable system for drug testing as they provide a closer model of cancer heterogeneity as compared to commercial cell lines ${ }^{22-25}$. Primary spheroid cultures were derived from both lung and colorectal cancer (CRC) patients, biobanked at early passages after isolation, characterized for common genetic mutations and validated for their ability to reproduce the histology of the original tumor in mouse xenografts ${ }^{25,26}$. We tested NanoFEN on twenty-one spheroid lines of CRC and six spheroid lines of lung cancer, the latter being representative of all the major histological tumor types (adenocarcinoma, squamous cell carcinoma, large cell neuroendocrine carcinoma). NanoFEN was able to induce death of colon and lung spheroids, with the latter showing a sensitivity related to tumor histotype (Fig. 2a and Supplementary Fig. 2a, b), although an analysis on a larger number of samples would be required to confirm this correlation. Notably, the sensitivity of colorectal spheroids to NanoFEN was independent of the presence of common mutations incompatible with anti-EGFR agents, suggesting that patients not eligible for targeted therapies could in principle benefit from NanoFEN treatment. NanoFEN was also tested on three ATCC CRC cell lines and nine ATCC lung cancer cell lines, where it induced cell death with higher IC50 as compared to primary spheroid lines (Fig. 2b and Supplementary Fig. 2a, b). We reasoned that the presence of $10 \%$ serum in the culture media of ATCC lines may account for their lower sensitivity to NanoFEN as compared to spheroid lines (which are grown in serumfree media). Thus, we treated ATCC cells with NanoFEN in the presence of $1 \%$ serum and noticed that the IC50 was affected in CRC cell lines but not in lung cancer cell lines (Supplementary Fig. 2a, b). Thus, the differential sensitivity of ATCC lines and of spheroid lines likely depends on a combination of culture conditions and of yet unidentified factors. Finally, we tested the effect of NanoFEN on primary cultures of melanoma, breast cancer, glioblastoma and sarcoma, and on ATCC lines of hematologic cancer cells. NanoFEN displayed a broad spectrum cytotoxic effect on all the cells tested and particularly on primary cultures, which displayed IC50 values ranging from 0.2 to $4.9 \mu \mathrm{M}$ (Supplementary Fig. 2c-g). These results suggest that NanoFEN interferes with mechanisms shared by multiple tumors to inhibit cell viability.

\section{In vivo antitumor efficacy of NanoFEN on lung and CRC xenografts}

We assessed the antitumor effects of NanoFEN compared to conventional chemotherapeutic agents on lung and CRC xenografts derived from four primary spheroid lines by monitoring tumor growth during treatment and the variation of tumor size after treatment withdrawal. NanoFEN and chemotherapy treatment significantly inhibited tumor progression in lung squamous carcinoma xenografts, lung adenocarcinoma xenografts (Fig. 3a, b left) and CRC xenografts, the latter with or without mutated KRAS (Fig. 3c, d left). After treatment interruption, xenografts were monitored for two additional weeks in order to monitor the rate of tumor regrowth. While xenografts treated with chemotherapy showed accelerated growth rates after treatment cessation, NanoFEN-treated xenografts grew significantly slower, suggesting that NanoFEN does not induce an enrichment of tumorigenic cells (Fig. 3a-d right). Finally, we investigated the feasibility of a prolonged treatment with NanoFEN in mice. Mice bearing lung cancer xenografts were treated with NanoFEN for 70 days, during which we observed an 

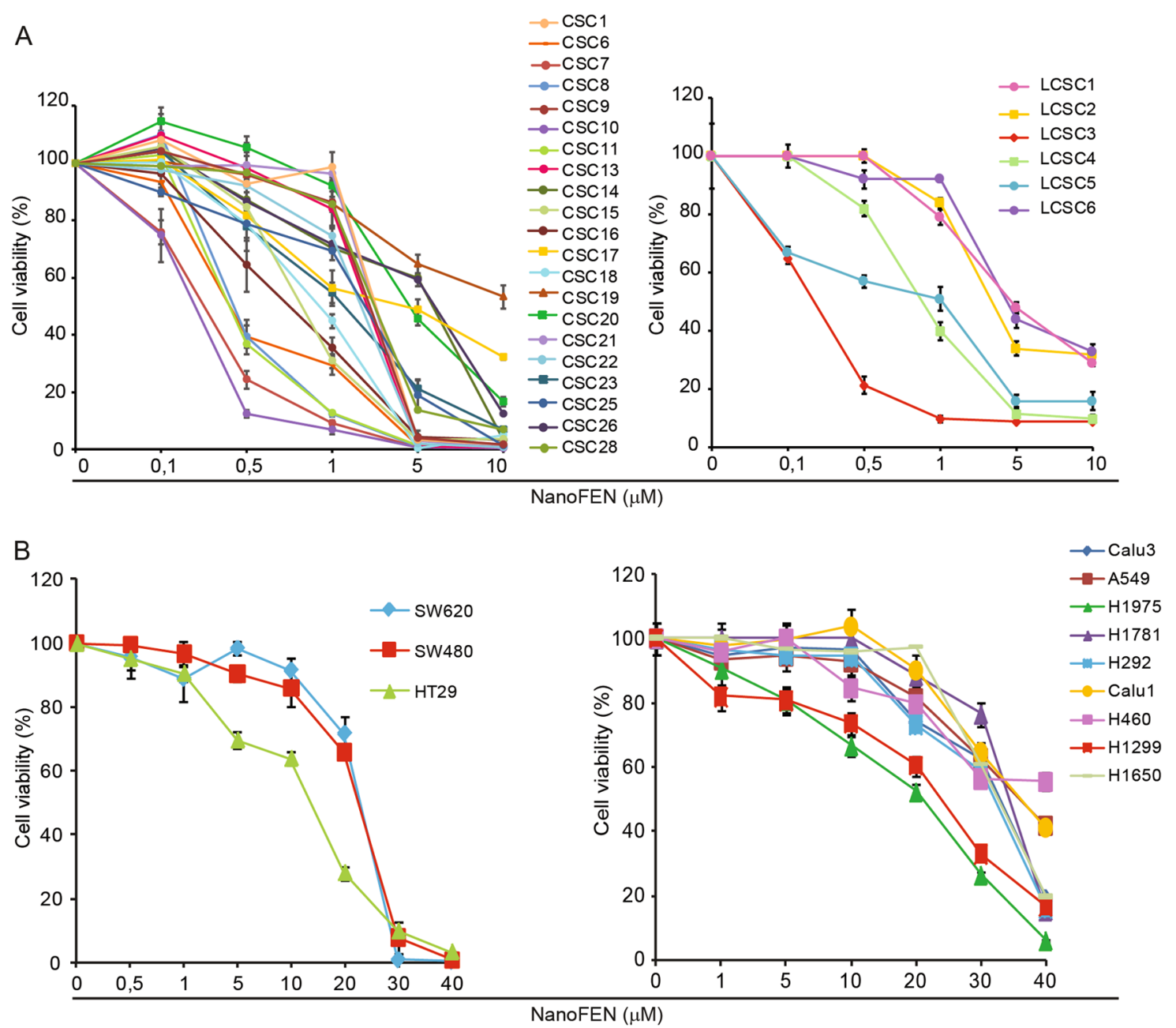

Fig. 2 Effects of NanoFEN on lung and colorectal cancer cells. a Cell viability of CRC spheroid cells (CSCs) (left) and lung cancer spheroid cells (LCSCs) (right) treated with NanoFEN at the indicated concentrations. b Cell viability of CRC commercial cell lines (left) and lung cancer commercial cell lines (right) treated with NanoFEN at the indicated concentrations. Values represent mean \pm SD of three independent experiments

extended arrest of tumor growth without any animal death or evident signs of toxicity (Supplementary Fig. 3a). Such observations, if confirmed in humans, could candidate NanoFEN as a compound suitable for long-term treatments such as those aimed at preventing tumor recurrence.

\section{Targeted phospho-proteomics on NanoFEN-treated primary cancer cells}

We aimed to generate a comprehensive picture of NanoFEN-induced changes in pathway activation by Reverse-Phase Protein Array (RPPA), a high-throughput technology based on the detection of proteins along with their post-traslational protein modifications, e.g., cleavage and phosphorylation ${ }^{27}$. To this end, we performed RPPA using a selection of 72 antibodies (Supplementary Table 1) on lung cancer spheroids left untreated or treated with NanoFEN at the IC50 dose. RPPA analysis revealed a NanoFEN-dependent modulation of multiple pathways involved in cell proliferation and biosynthesis, cell cycle inhibition, cell death and stemness. In detail, treatment with NanoFEN was associated with down-modulation of multiple components of the mTOR pathway, i.e., decreased levels of phospho-mTOR, phospho-eIF4G and phospho-4EBP1 or proliferation pathway, i.e., decreased levels of phospho-ERK $1 / 2$; induction of cell death and DNA damage response, i.e., increased amounts of cleaved Caspase 7, cleaved PARP and phospho-H2A.XY and a decrease of cell cycle-related factors, i.e., phospho-Aurora A/B/C, phospho-CDC2, phospho-PLK1, phospho$\mathrm{CDC} 25 \mathrm{c}$, phospho-Histone $\mathrm{H} 3$ as well as increased levels of p16 and of phospho-p38 MAPK, accompanied by a 
A

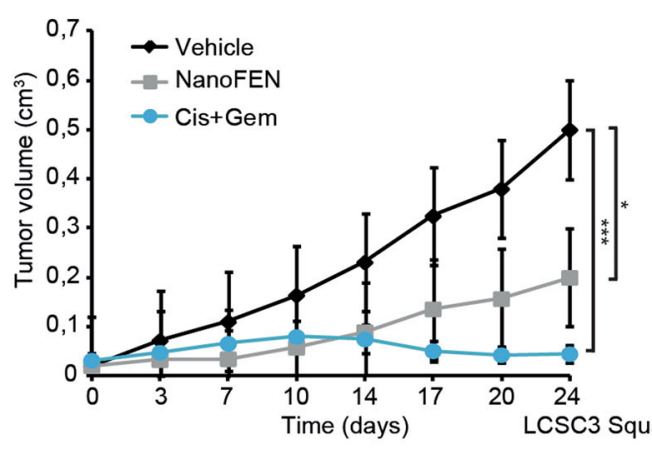

B

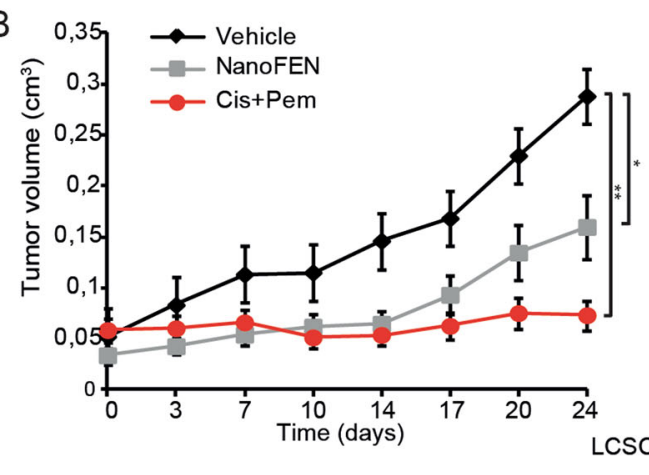

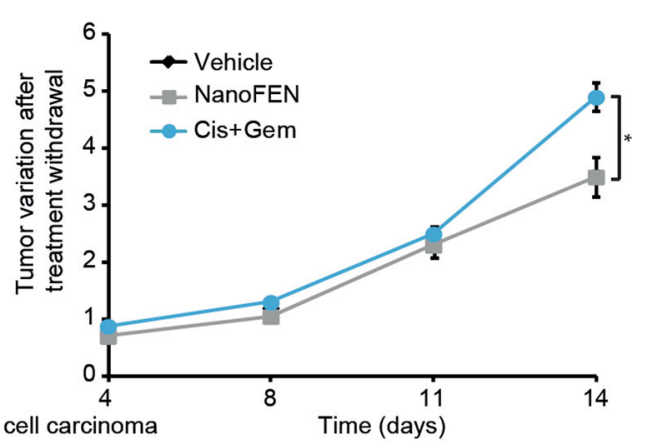

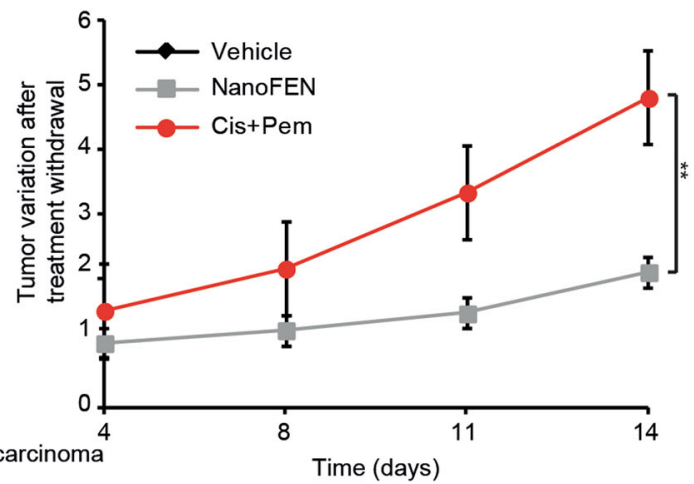

C
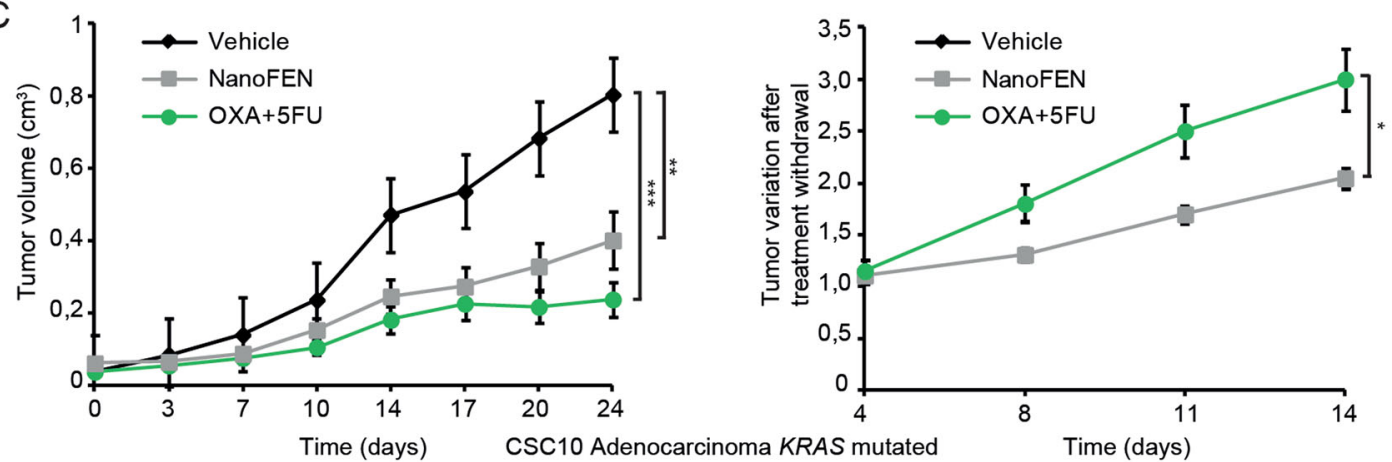
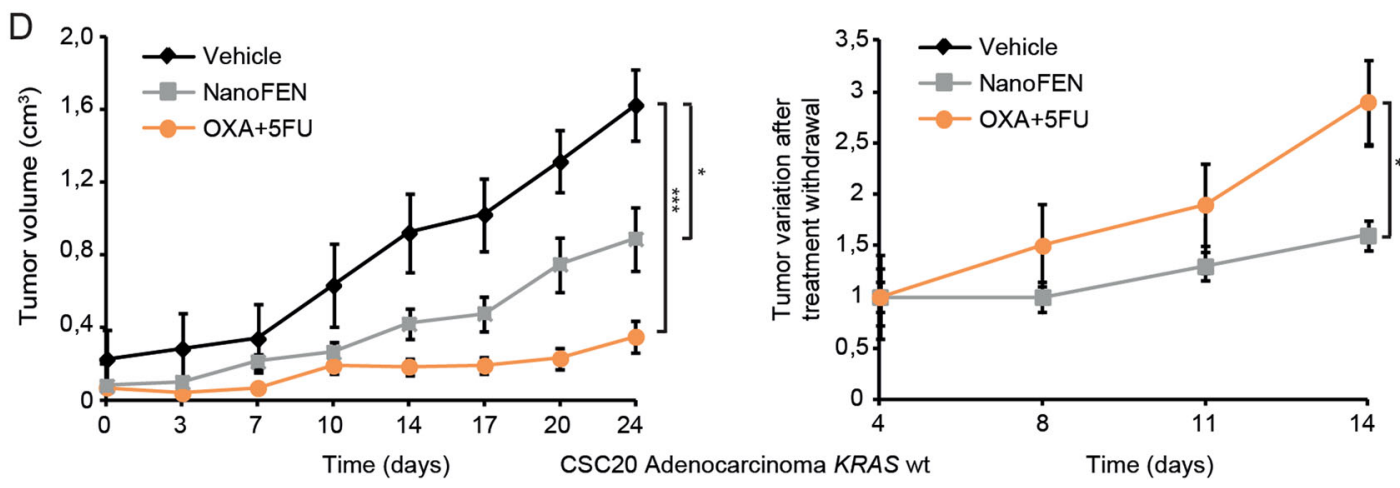

Fig. 3 (See legend on next page.) 
(see figure on previous page)

Fig. 3 Antitumor efficacy of NanoFEN on lung and CRC xenografts. a Left panel, volume of xenografts derived from squamous cell carcinoma lung cancer spheroid cell line 3 (LCSC3) treated with vehicle (black diamonds), NanoFEN (grey squares) and cisplatin (Cis) plus gemcitabine (Gem) (blue circles); right panel, tumor variation after treatment withdrawal. b Left panel, volume of xenografts derived from adenocarcinoma lung cancer spheroid cell line 5 (LCSC5) treated with vehicle (black diamonds), NanoFEN (grey squares) and Cis plus pemetrexed (Pem) (red circles); right panel, tumor variation after treatment withdrawal. c Left panel, volume of xenografts derived from adenocarcinoma colorectal spheroid cell line 10 (CSC10) KRAS mutated treated with vehicle (black diamonds), NanoFEN (grey squares) and oxaliplatin (OXA) plus 5-fluorouracil (5FU) (green circles); right panel, tumor variation after treatment withdrawal. $\mathbf{d}$ Left panel, volume of xenografts derived from adenocarcinoma colorectal spheroid cell line 20 (CSC20) KRAS wild type, treated with vehicle (black diamonds), NanoFEN (grey squares) and OXA plus 5FU (orange circles); right panel, tumor variation after treatment withdrawal. Values represent the mean \pm SEM of three independent experiments. ${ }^{*} P<0.05 ;{ }^{* *} P<0.01 ;{ }^{* * *} P<0.001$ from one-way ANOVA and Bonferroni post-test

decrease of the stemness-related factor ALDH (Fig. 4a-c). Altogether, these results indicate that NanoFEN affects multiple cellular functions, whose readout endpoints underwent individual validation, as detailed in the following sections.

\section{Cell cycle and stemness inhibition by NanoFEN in lung and colorectal cancer spheroid cultures}

RPPA results indicated that NanoFEN modulated cell cycle-related factors (Fig. 5a), potentially resulting in growth slowdown and/or arrest. We confirmed the effects of NanoFEN on cell cycle factors by validating the increase in total p16 levels and phospho-p38 MAPK, and the decrease in phospho-Aurora A-B-C and phosphoPLK1 by immunoblotting in NanoFEN-treated lung and CRC spheroids (Fig. 5b). Then, we investigated whether NanoFEN was able to induce growth arrest through the use of the mVenus $\mathrm{p} 27 \mathrm{~K}^{-}$construct, which identifies with yellow-green fluorescence the cells in G0 phase of the cell cycle and in G0/G1 transition ${ }^{28}$. Lung and colorectal spheroids were transduced with mVenus $\mathrm{p} 27 \mathrm{~K}^{-}$, treated with NanoFEN and evaluated by flow cytometry. Upon a $48 \mathrm{~h}$ treatment, NanoFEN significantly increased the number of Venus ${ }^{+}$cells as compared with control cells indicating a higher number of G0 and G0/G1 transition cells, whereas chemotherapy strongly decreased Venus levels (Fig. 5c), indicating that in these conditions the two treatments have opposite effects of on the cell cycle. The decrease in ALDH levels observed in RPPA experiments prompted us to investigate whether NanoFEN would decrease tumor stem cell content (Fig. 5d). Aldefluor activity increased with chemotherapy, according to several studies showing that chemotherapeutic agents induce a population of drug-tolerant stem cells ${ }^{29}$, but decreased in NanoFEN-treated lung spheroids (Fig. 5e and Supplementary Fig. 3b). In line with this observation, we detected a decrease of genes related to stemness and selfrenewal (NANOG, SOX2, POU5F1) upon NanoFEN treatment and a parallel increase in chemotherapy-treated cells (Fig. 5f) in lung spheroids. The TOP-GFP system provides a functional evaluation of stem cell content in
CRC by recapitulating the expression of the Wnt target TCF linked to $\mathrm{GFP}^{30}$. Thus, we investigated TOP-GFP expression in vitro and in vivo upon NanoFEN treatment in cells transduced with a TOP-GFP.mCherry vector. NanoFEN significantly reduced TOP-GFP expression in vitro upon a short-term treatment (Fig. $5 \mathrm{~g}$ ). In vivo treatment with chemotherapy or NanoFEN of xenografts obtained with TOP-GFP-transduced CRC cells resulted, as expected, in decreased tumor growth as compared to controls (Fig. 5h). Importantly, analysis of TOP-GFP content at the end of the treatment in relation to tumor volume showed that the proportion of TOP-GFP ${ }^{+}$cells increased upon chemotherapy but decreased upon NanoFEN treatment (Fig. 5i and Supplementary Fig. 3c). Altogether, these observations indicate that NanoFEN is able to induce a quiescent state and to decrease the content of cells with stem-like features in lung and CRC.

\section{Cell death pathways activated by NanoFEN in primary lung and CRC spheroid cells}

As shown by RPPA analyses, NanoFEN treatment affects several factors involved in DNA damage, cellular stress and apoptosis. To gain further insights into deathrelated events activated by NanoFEN we validated and extended RPPA results by immunoblotting in lung and colon spheroids, thus confirming NanoFEN-mediated caspase activation and cleavage of apoptotic substrates (Fig. 6a, b and Supplementary Fig. 4a). Pre-treatment with the pan-caspase inhibitor zVAD-fmk (zVAD) did not substantially inhibit NanoFEN-induced toxicity, suggesting that caspase activity is dispensable for NanoFENinduced death (Fig. 6c), similarly to what has been described for the classical formulation of fenretinide. Because reactive oxygen species (ROS) generation has been reported to occur upon fenretinide treatment in different cellular systems ${ }^{31}$, we next investigated the production of ROS in NanoFEN-treated lung and colon spheroids. We detected a moderate increase in ROS levels in both lung and CRC cells, as shown by $\mathrm{H}_{2}$-DCFDA staining (Fig. 6d). The ROS scavenger $\mathrm{N}$-acetyl-cysteine (NAC) partially rescued NanoFEN-induced death in both 


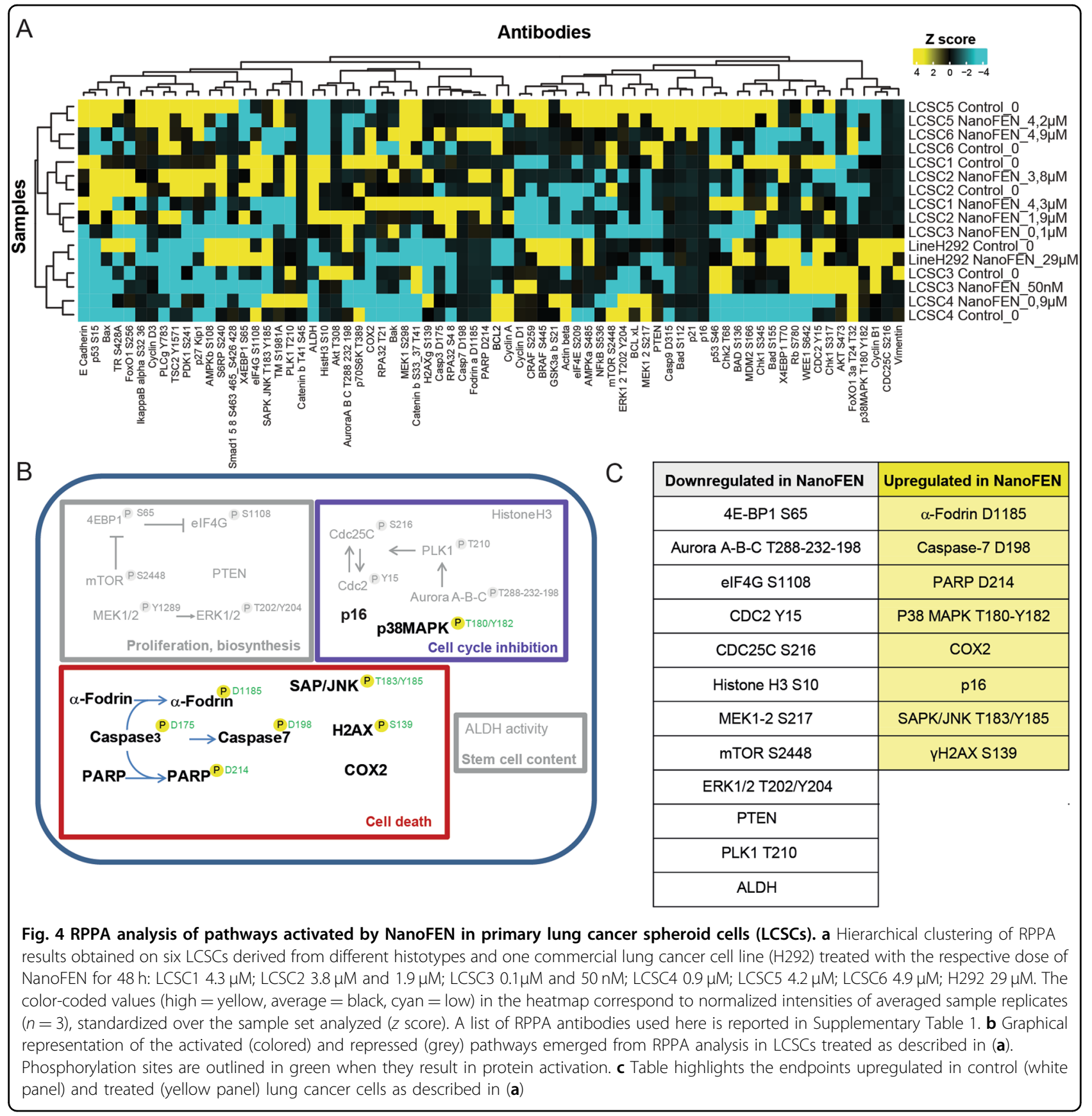

lung and colon CSCs and reduced ROS levels (Fig. 6e and Supplementary Fig. 4b) suggesting that ROS generation plays a role in NanoFEN cytotoxicity, in line to what reported in leukemic stem cells ${ }^{32}$. Since the repression of the mTOR pathway that emerged from RPPA analyses is known to promote autophagy, we investigated the presence of autophagy-related markers in lung and CRC spheroids treated in vitro with NanoFEN. Interestingly, we found in the majority ( 3 out of 4 ) of the spheroid lines tested an increased expression of both LC3 and its phosphatidylethanolamine conjugate (LC3-II), which is recruited to autophagosomal membranes ${ }^{33}$ (Fig. 6f and Supplementary Fig. 4c). We also observed that the autophagy inhibitor 3-MA was able to counteract NanoFEN-induced death (Fig. 6g), suggesting that autophagy contributes to NanoFEN-induced death in both lung and CRC cells.

\section{Modulation of cellular metabolism by NanoFEN}

Fenretinide treatment of cancer cells has been previously associated with the production of lipid second messengers including ceramide, gangliosides (both 
A

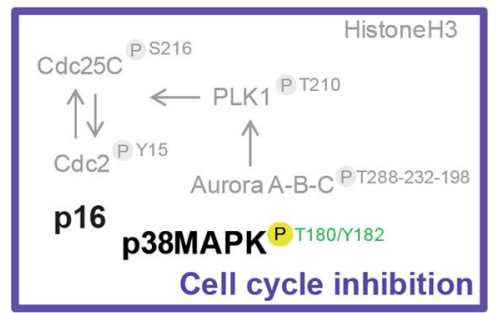

C

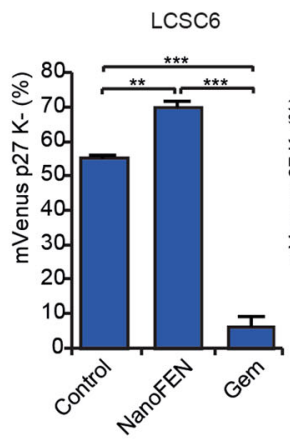

D

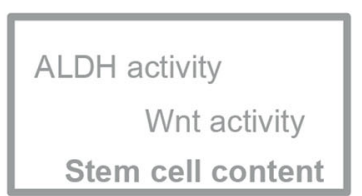

G

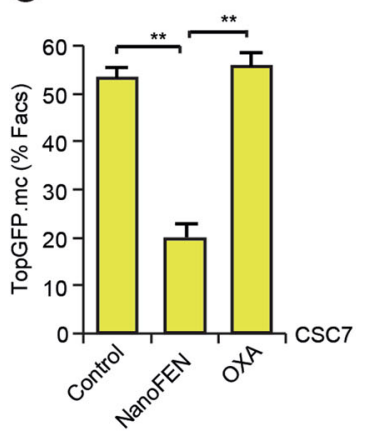

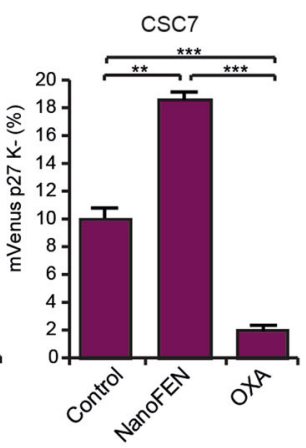

E

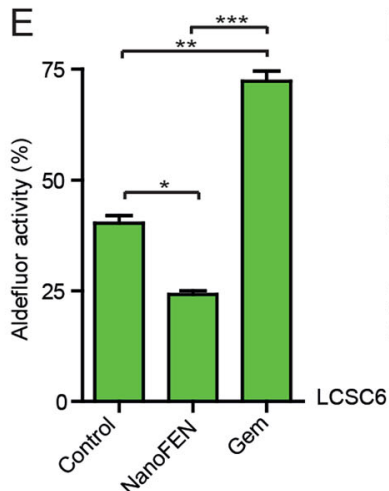

$\mathrm{H}$

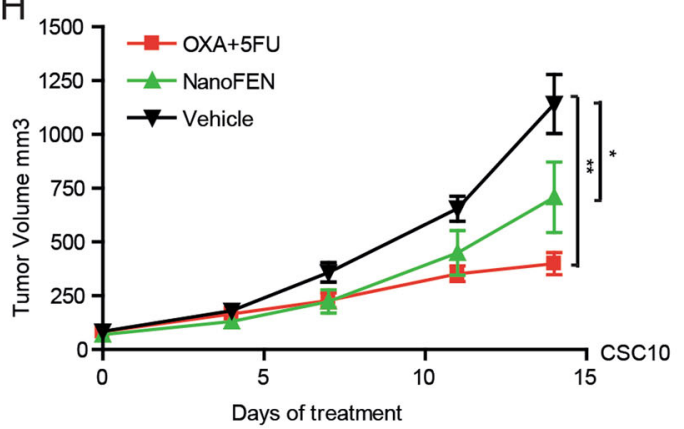

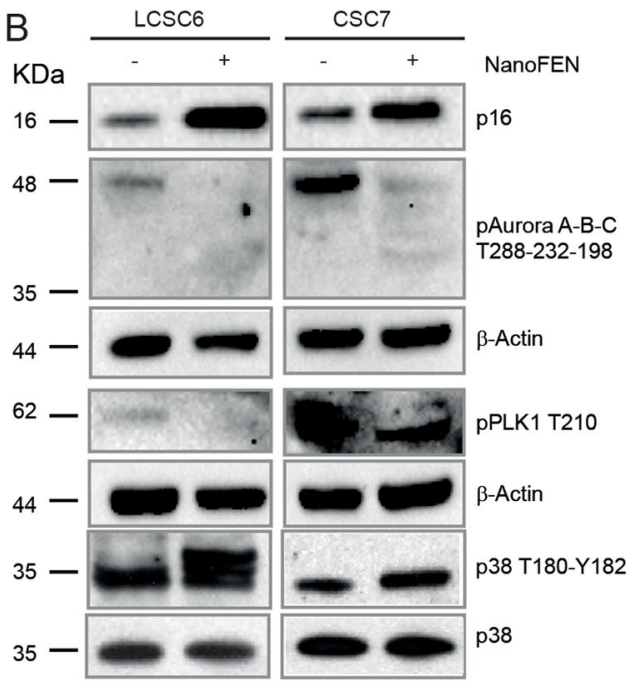

F $\quad$ NANOG $\square$ SOX2 $\square$ POUSF1

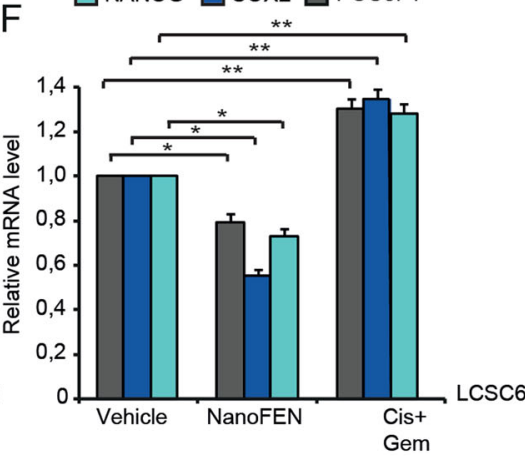

I

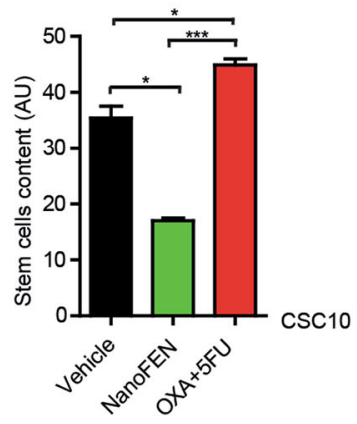

Fig. 5 (See legend on next page.)

involved in apoptosis induction) and dihydroceramide $^{34,35}$. Moreover, RPPA results showed a global inhibition of the mTOR pathway (Fig. 7a) that was subsequently confirmed by immunoblotting (Fig. 7b and Supplementary Fig. 5a), thus linking NanoFEN mechanism of action to metabolism, biosynthesis and lipid signaling networks. We assessed the effect of NanoFEN on cellular lipids by liquid chromatography-mass spectrometry (LC-MS) in lung and CRC spheroid cultures, revealing a massive accumulation of $\mathrm{d} 18$ dihydroceramide in treated cells (Fig. 7c and Supplementary Fig. 5b) as compared to other ceramides (Supplementary Fig. 5c-g). 
(see figure on previous page)

Fig. 5 Effects of NanoFEN on cell cycle and stem-like features in vitro and in vivo on lung cancer spheroid cells (LCSCs) and CRC spheroid cells (CSCs). a Schematic representation of cell cycle regulators activated (black) or repressed (grey) arised from RPPA analysis of LCSCS treated with NanoFEN as described in Fig. 4a. b Immunoblot analysis of cell cycle regulators phospho-Aurora A-B-C, phospho-PLK1, p38, phospho-p38 and p16 on LCSC6 (left panel) and CSC7 (right panel) untreated and treated with NanoFEN at the IC50 concentration ( $4.9 \mu \mathrm{M}$ and $0.2 \mu \mathrm{M}$ respectively) for $48 \mathrm{~h}$. c Flow cytometry analysis of mVenus p27K- -transduced LCSC6 (left panel) and CSC7 (right panel) untreated and treated with NanoFEN at the IC50 dose $\left(4,9 \mu \mathrm{M}\right.$ and $0,2 \mu \mathrm{M}$ respectively) for $48 \mathrm{~h}$, Gem $25 \mu \mathrm{M}$ and OXA $10 \mu \mathrm{M}$. Values represent mean \pm SD of three independent experiments. ${ }^{* *} P<$ 0.01 ; ${ }^{* *} P<0.001$ from two-tailed $t$-test. $\mathbf{d}$ Schematic representation of stem-like factors (repressed in grey) emerged from RPPA analysis of lung CSCS treated as described in Fig. 4a. e Cytofluorimetric analysis of Aldefluor activity on LCSC6 treated with NanoFEN at IC50 dose and Gemcytabine $25 \mu \mathrm{M}$ for $48 \mathrm{~h}$. Values represent mean \pm SD of three independent experiments. ${ }^{* *} P<0.01$; ${ }^{* *} P<0.001$ from two-tailed $t$-test. $\mathbf{f}$ qRT-PCR analysis of indicated genes on whole tumor lysates derived from LCSC6 xenografts, untreated or treated with NanoFEN $30 \mathrm{mg} / \mathrm{Kg} / \mathrm{week}$ and Cis $3 \mathrm{mg} / \mathrm{Kg} / \mathrm{week}$ and Gem $60 \mathrm{mg} / \mathrm{Kg} /$ week (Cis $+\mathrm{Gem})$. Values represent mean $\pm \mathrm{SD}$ of three independent experiments. ${ }^{*} P<0.05 ;{ }^{*} P<0.01$ from two-tailed $t$-test. $\mathbf{g}$ Flow cytometry analysis of TOP-GFP.mc-transduced CSC7, untreated and treated with NanoFEN at the IC50 dose $0.2 \mu \mathrm{M}$ and OXA $10 \mu \mathrm{M}$ for $48 \mathrm{~h}$. Values represent mean $\pm S D$ of three independent experiments. ${ }^{*} P<0.01$ from two-tailed $t$-test. $\mathbf{h}$ Volume of xenografts derived from CSC10 transduced with TOP-GFP.mc untreated (black downward triangles) or treated with vehicle or treated NanoFEN $30 \mathrm{mg} / \mathrm{Kg} /$ week (green upward triangles) and OXA $5 \mathrm{mg} / \mathrm{Kg} /$ week plus $5 F U 12.5 \mathrm{mg} / \mathrm{Kg} /$ week (red squares). Values represent mean \pm SEM three independent experiments. ${ }^{*} P<0.05$; ${ }^{* *} P<0.01$ from one-way ANOVA and Bonferroni post-test. i Total stem cell content in tumor xenografts obtained as in (h), relative to the experiment shown in Supplementary Fig. 3c. Values were calculated as tumor weight $\times$ percentage of TOP-GFP.mc positive cells/100. Values represent mean \pm SD of three independent experiments. ${ }^{*} P<0.05 ;{ }^{* *} P<0.001$ from two-tailed $t$-test

Dihydroceramide production was previously shown to elicit multiple biological effects in cancer cells such as G0/ G1 cell cycle arrest, impairment of mitochondrial function and autophagy induction ${ }^{36-38}$, corresponding to the biological effects observed in NanoFEN-treated cells. These observations suggest that NanoFEN (as was previously shown for fenretinide) targets dihydroceramide desaturase (DES), thus inducing dihydroceramide accumulation. Accordingly, we observed a strong increase of sphinganine (SA, the lipid mediator upstream of DES) both in vitro in NanoFEN-treated lung and CRC cells (Fig. 7d) and in vivo in lung cancer xenografts (Supplementary Fig. 6a). In order to determine the functional relevance of dihydroceramide production in NanoFEN-treated cells we used the serine palmitoyltransferase inhibitor myriocin, which blocks de novo sphingolipid biosynthesis ${ }^{39}$. Pretreatment of spheroid lines with myriocin partially protected cells from NanoFEN-induced death, indicating an active role of the sphingolipid pathway in mediating NanoFEN effects (Fig. 7e). In line with an important role of dihydroceramide in mediating NanoFEN effects, SA and the DES inhibitor GT11 induced a dose-dependent viability decrease in lung cancer spheroids (Supplementary Fig. 6b). By contrast, the glucosylceramide synthase inhibitor 1-phenyl-2-palmitoylamino-3-morpholino-1propanol (PPMP) was unable to block NanoFEN-induced death, indicating that the cytotoxic effects of NanoFEN are determined by the accumulation of dihydroceramide rather than by glucosylceramide/ceramide formation (Fig. 7f). Finally, we ought to determine whether the sphingolipid pathway would cooperate with repression of the mTOR pathway in mediating NanoFEN-induced toxicity. To this aim, we mimicked the effect of NanoFEN by treating spheroids with both sphinganine and GT11 at low doses. Additional treatment with the mTOR pathway inhibitor rapamycin significantly potentiated the toxic effects of the SA/GT11 combination, indicating a cooperative effect of the two pathways in inducing cancer cell death (Fig. 7g and Supplementary Fig. 6c). Western blot analysis of key factors implicated in mTOR signaling showed that the combined treatment with SA/GT11/ rapamycin effectively inhibited the mTOR pathway and recapitulated NanoFEN-related events (Fig. 7h).

\section{Discussion}

In accordance to their multifaceted role in cell proliferation, differentiation and death, retinoids have long been studied for their chemopreventive and chemotherapeutic effects in cancer patients, although their mechanism of action has not been completely elucidated. One of the most promising retinoid derivatives is fenretinide, which has been extensively investigated in phase IIII clinical trials, revealing long-term safety and tolerability but modest anticancer effects $3,4,7-15,21,40-43$. The scarce efficacy of fenretinide in previous clinical studies is related to its low bioavailability, as even high-dose schedules and multiple administrations did not provide plasma concentrations sustained over time at the levels required for a therapeutic activity ${ }^{3,8,10-13}$. We report here that a nanoencapsulated form of fenretinide (previously modified through a salification procedure in order to increase complexation effectiveness) has an enhanced solubility and reaches significantly higher plasma levels than formulations previously used in clinical trials. The mechanism of action of NanoFEN was analyzed in primary spheroid cultures of lung and $\mathrm{CRC}$, thus providing a comprehensive picture of protein and lipid pathways activated in cancer cells. The results emerging from proteomic and lipidomic analyses revealed a complex set of molecular events activated by NanoFEN, resulting in a 


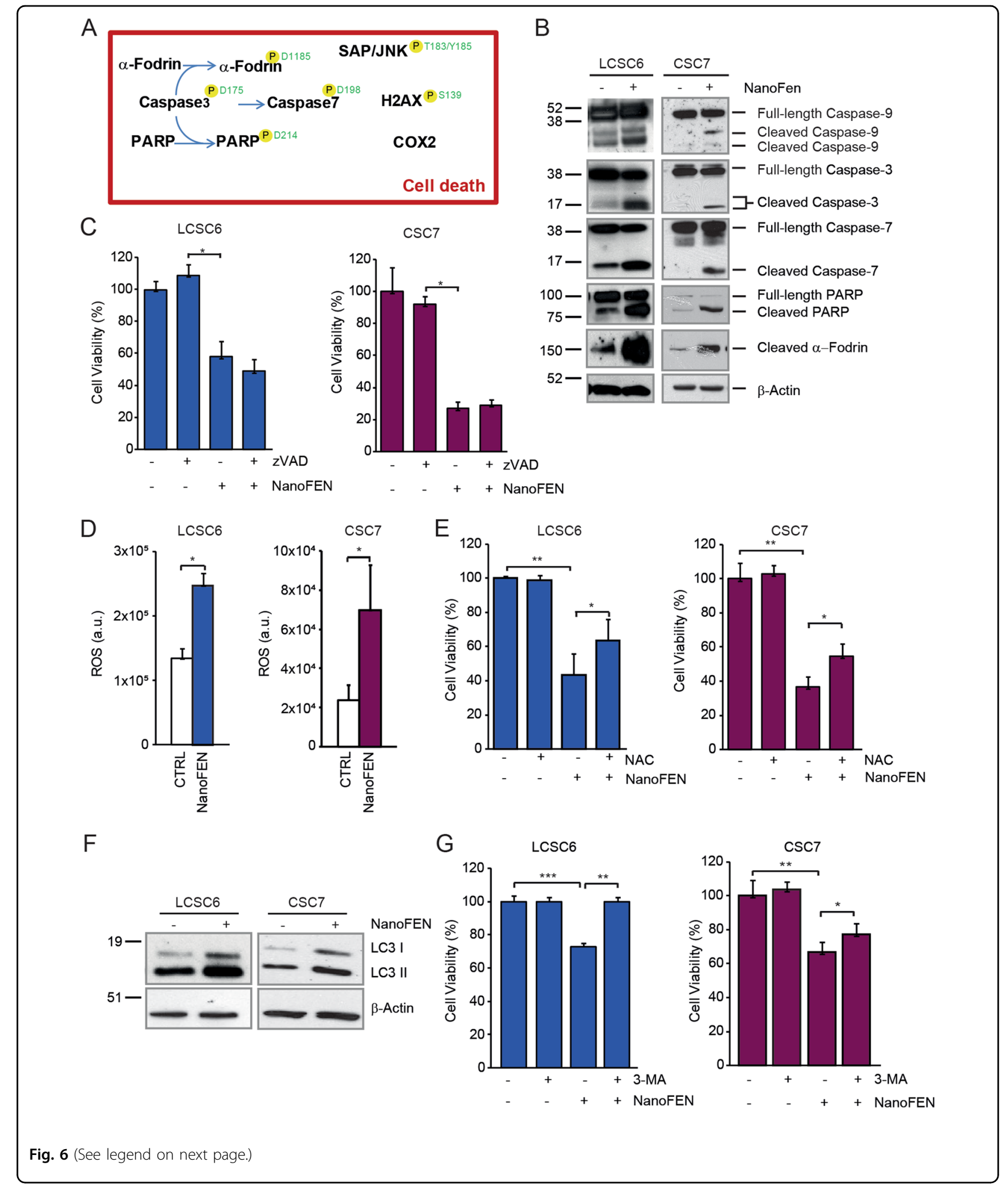

B

broad landscape of functional effects ranging from cell death (with features of both autophagy and apoptosis) to cell cycle arrest and to a generalized metabolic repression mediated by inhibition of the mTOR pathway and massive production of dihydroceramide. The effect of NanoFEN in inducing growth arrest/quiescence is particularly interesting in view of a potential use of this compound in chemopreventive settings. In fact, maintaining tumor cells 


\begin{abstract}
(see figure on previous page)
Fig. 6 Analysis of cell death pathways activated by NanoFEN in lung cancer spheroid cells (LCSCs) and CRC spheroid cells (CSCs). a Schematic representation of RPPA endpoints involved in DNA damage, cell stress and apoptosis activated (black) in LCSCS treated with NanoFEN as described in Fig. 4a. Phosphorylation sites are outlined in green when they result in protein activation. $\mathbf{b}$ Immunoblot analysis of cell death-related proteins Caspase-9, 3, and 7, PARP and a-fodrin on LCSC6 (left panel) and CSC7 (right panel) untreated and treated with NanoFEN at IC50 dose (4.9 and $0.2 \mu \mathrm{M}$, respectively) for $48 \mathrm{~h}$. c Cell viability of LCSC6 (left panel) and colon CSC7 (right panel) pretreated with zVAD $40 \mu \mathrm{M}$ and then with NanoFEN at IC50 dose (4.9 and $0.2 \mu \mathrm{M}$, respectively) for $48 \mathrm{~h}$. Values represent mean \pm SD of three technical replicates of a representative experiment of two biological replicates. ${ }^{*} P<0.05$ from two-tailed $t$-test. $\mathbf{d}$ Production of reactive oxygen species (ROS) was measured by flow cytometer in LCSC6 (left panel) and CSC7 (right panel) untreated or treated with NanoFEN (4.9 and $0.2 \mu \mathrm{M}$, respectively) for $24 \mathrm{~h}$ and then with $5 \mu \mathrm{M}$ of CM- $\mathrm{H}_{2} \mathrm{DCFDA}$. Values represent mean \pm SD of three independent experiments. ${ }^{*} P<0.05$ from two-tailed $t$-test. e Cell viability of LCSC6 (left panel) and CSC7 (right panel) pretreated with $\mathrm{N}$-acetyl-cysteine (NAC) $2 \mathrm{mM}$ for $1 \mathrm{~h}$ and after with NanoFEN for $48 \mathrm{~h}$ ( 4.9 and $0.2 \mu \mathrm{M}$ respectively). Values represent mean \pm SD of three technical replicates of a representative experiment. ${ }^{*} P<0.05$; ${ }^{*} P<0.01$ from two-tailed $t$-test. $\mathbf{f}$ Immunoblot analysis of autophagyrelated marker LC3 I-II on LCSC6 (left panel) and CSC7 (right panel) untreated and treated with NanoFEN at IC50 dose (4.9 and $0.2 \mu \mathrm{M}$, respectively) for $24 \mathrm{~h} . \mathbf{g}$ Cell viability of LCSC6 (left panel) and CSC7 (right panel) treated with 3-Methyladenine (3-MA) $5 \mathrm{mM}$ plus NanoFEN $(4.9$ and $0.2 \mu \mathrm{M}$, respectively) for $24 \mathrm{~h}$. Values represent mean \pm SD of a three technical replicates of a representative experiment. ${ }^{*} P<0.05$; ${ }^{* *} P<0.01 ;{ }^{* * *} P<0.001$ from two-tailed $t$-test
\end{abstract}

(particularly those disseminated in distant organs) in a prolonged and stable quiescent state is progressively emerging as a potential therapeutic strategy aimed at preventing tumor relapse ${ }^{44}$. In line with this hypothesis, a combination of all-trans retinoic acid and 5-azacitidine has been reported to induce a quiescent state in bone marrow-disseminated cancer cells, supporting a role of retinoids in promoting a long-term metastasis-free condition $^{45-47}$ and is currently being investigated in a clinical trial (NCT03572387). If confirmed in additional preclinical and clinical studies, the low toxicity of NanoFEN would allow prolonged treatment schedules aimed at avoiding the reactivation of disseminated tumor cells and subsequent tumor relapse, particularly in cancers subject to late recurrences such as those of breast and prostate. Moreover, it would be interesting to investigate the effect of NanoFEN in retinoid-sensitive tumors such as some hematological tumors as well as neuroblastoma, where the increased bioavailability of NanoFEN may allow more effective treatment schedules as compared to previous clinical trials. In summary, the results reported in this study indicate NanoFEN as a bioavailable fenretinide formulation with broad-range inhibitory effects on cancer cells that may find a future use in several clinical settings.

\section{Materials and methods}

\section{Cells, tissues, mice, antibodies, and reagents}

Colon and lung cancer specimens were obtained from patients undergoing surgical resection upon informed consent and approval by the Sapienza-Policlinico Umberto I Ethical Committee (RIF.CE: 410717/10/ 2016). Spheroid cultures were established and validated as previously described ${ }^{48,49}$. All the commercial cancer cell line were purchased from the American Type Culture Collection (ATCC, Manassas, VA, USA) or Sigma-Aldrich (St. Louis, MO, USA) and cultured following manufacturer's instructions. Fenretinide, N-(4-Hydroxyphenyl) retinamide (code 65646-68-6) was purchased from Olon
(Milan, Italy). 2-hydroxypropyl- $\beta$-cyclodextrin (average $\mathrm{Mw} \sim 1380$ ), potassium hydroxide, 1-octanol, $\mathrm{D}_{2} \mathrm{O}$, DMSO-d6 were purchased from Sigma-Aldrich. Antibodies used for RPPA and western blot were listed in Supplementary Table 1. Oxaliplatin and 5-fluorouracil were from Teva Italia (Milano, Italy) gemcitabine and cisplatin were from Selleckchem (Houston, TX, USA). Aldefluor assay was from STEMCELL Technologies (Vancouver, Canada). Myriocin was purchased from ENZO Life Science (New York, NY, USA), N-[(1R,2S)-2hydroxy-1-hydroxymethyl-2-(2-tridecyl-1-cyclopropenyl) ethyl]octanamide (GT11) and Sphinganine from Avanti Polar Lipids (Alabaster, AL, USA), Rapamycin from Selleckchem, z-Val-Ala-DL-Asp-fluoromethylketone (zVAD) from BACHEM (Torrance, CA, USA), DL-threo-1-Phenyl-2-hexadecanoylamino-3-morpholino-propanol-HCL (PPMP) from Matreya (State College, PA, USA), NAcetyl-L-Cysteine (NAC) was purchased from SigmaAldrich, 5-chloromethylfluoresceindiacetate (CMFDA) was purchased from Molecular Probes (Eugene, OR, USA). Anti-LC3 and anti- $\beta$-Actin antibodies were purchased from Sigma-Aldrich; phospho-Aurora A (Thr288)/ Aurora B (Thr232)/Aurora C (Thr198) (D13A11), phospho-PLK1, phospho-S6 ribosomal protein (Ser240/244) and total S6 ribosomal protein (5G10), caspase3, caspase7, caspase9, PARP, fodrin cleaved, phospho-p38 MAP kinase (Thr180/Tyr182), mTOR, phospho-mTOR (Ser2448), 4EBP1(53H11), phospho-4E-BP1(Ser65) antibodies were from Cell Signaling Technology (Danvers, MA, USA). Anti-CDKN2A (p16) was from Biolegend (London, United Kingdom). Secondary anti-mouse and anti-rabbit antibodies coupled to horseradish peroxidase were from Bio-Rad (Hercules, CA, USA).

\section{Preparation of fenretinide 2-hydroxypropyl- $\beta$-cyclodextrin complex (NanoFEN)}

The first step of NanoFEN preparation involved fenretinide salification to raise its aqueous solubility to the 


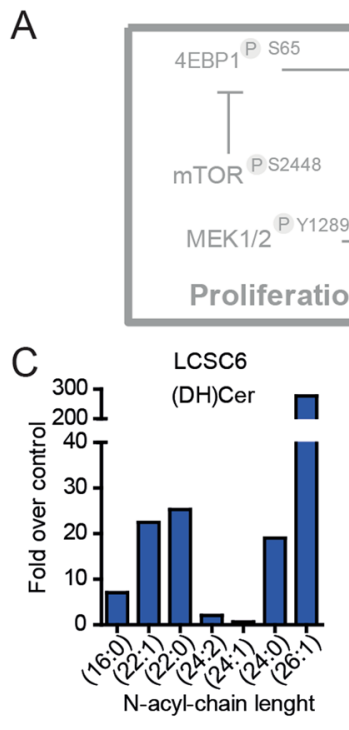

D
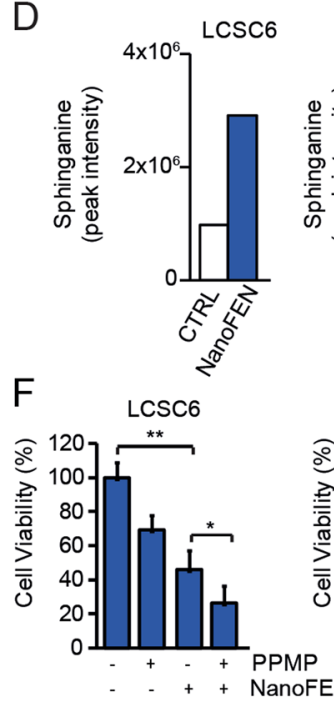

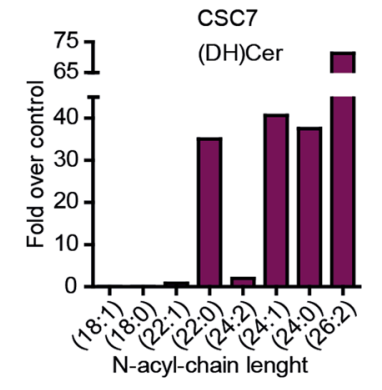

E

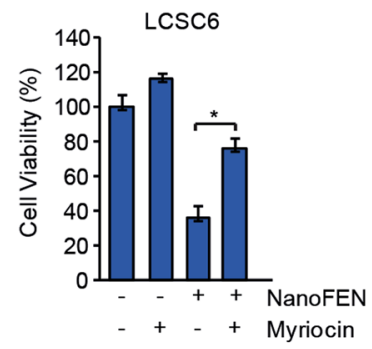

$\mathrm{G}_{140} \quad \operatorname{LCsc} 6$

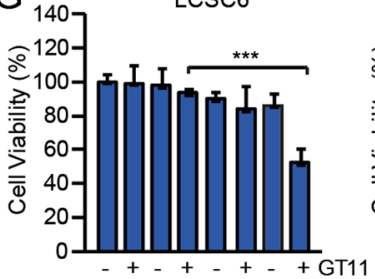

-++-++ SA

-+++ SA
++++ Rapamycin
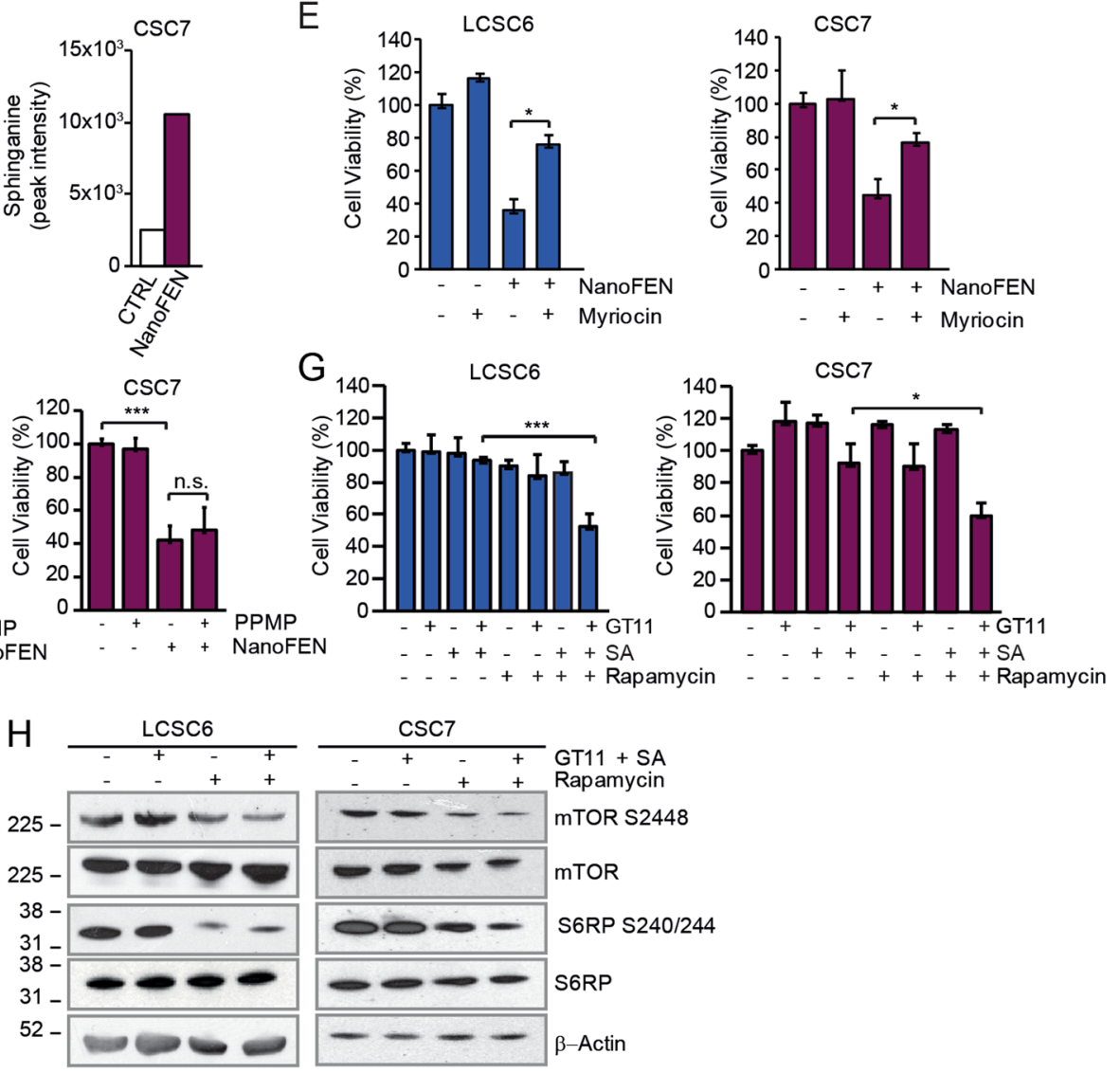

Fig. 7 (See legend on next page.)

extent required for complexation with cyclodextrins. Salification was carried out by dissolving fenretinide ( $2 \mathrm{~mol}$ in $20 \mathrm{~mL}$ ethanol containing a stoichiometric amount of potassium hydroxide. The solvent was removed in a rotary evaporator to obtain a solid red residue of fenretinide potassium salt (4-HPRK). The salt 
(see figure on previous page)

Fig. 7 NanoFEN inhibits cellular metabolism in lung cancer spheroid cells (LCSCs) and CRC spheroid cells (CSCs). a Schematic representation of RPPA results of proliferative and biosynthetic pathways repressed (grey) in LCSCs treated with NanoFEN. $\mathbf{b}$ Immunoblot analysis of proteins involved in metabolic process on LCSC6 (left panel) and CSC7 (right panel) untreated and treated with NanoFEN at IC50 dose (4.9 and $0.2 \mu \mathrm{M}$, respectively) for $48 \mathrm{~h}$. c Graphs represent NanoFEN effects on lipid metabolism by liquid chromatography-mass spectrometry (LC-MS) on LCSC6 (left panel) and CSC7 (right panel). Values represents the fold over control of treated cells with NanoFEN ( 4.9 and $0.2 \mu \mathrm{M}$, respectively) for $24 \mathrm{~h}$. d Sphinganine peak intensity in LCSC6 (left panel) and CSC7 (right panel) treated with NanoFEN ( 4.9 and $0.2 \mu \mathrm{M}$, respectively) for $24 \mathrm{~h}$. e Cell viability of LCSC6 (left panel) and CSC7 (right panel) treated with Myriocin $1 \mu \mathrm{M}$ plus NanoFEN (4.9 and $0.2 \mu \mathrm{M}$, respectively) for $48 \mathrm{~h}$. Values represent mean \pm SD of three independent experiments. ${ }^{*} P<0.05$, from two-tailed t-test. $\mathbf{f}$ Cell viability of LCSC6 (left panel) and CSC7 (right panel) treated with glucosylceramide synthase inhibitor PPMP $10 \mu \mathrm{M}$ plus NanoFEN (4.9 and $0.2 \mu \mathrm{M}$, respectively) for $48 \mathrm{~h}$. Values represent mean \pm SD of three independent experiments. ${ }^{*} P<0.05$, from two-tailed $t$-test. $\mathbf{g}$ Cell viability of LCSC6 (left panel) and CSC7 (right panel) treated with dihydroceramide desaturase inhibitor (GT11) $0.5 \mu \mathrm{M}$ plus sphinganine (SA) $2 \mu \mathrm{M}$ plus mTOR pathway inhibitor Rapamycin $10 \mathrm{nM}$ for $48 \mathrm{~h}$. Values represent mean \pm SD of three independent experiments. ${ }^{*} P<0.05$; ${ }^{* *} P<0.001$, from two-tailed $t$-test. $\mathbf{h}$ Immunoblot analysis of mTOR pathway components mTOR, phospho-mTOR, S6RP and phospho-S6RP on LCSC6 (left panel) and CSC7 (right panel) treated as in (g)

was physically mixed with 2-hydroxypropyl$\beta$-cyclodextrin at 1:10 w:wsalt:cyclodetrin ratio and grinded to homogeneity. The physical mixture obtained was dispersed in $30 \mathrm{~mL}$ water, stirred at room temperature $6 \mathrm{~h}$ in the dark and subsequently left at $4{ }^{\circ} \mathrm{C}$ overnight. After this period the suspension was stirred again for $2 \mathrm{~h}$ at room temperature, diluted with $20 \mathrm{~mL}$ water and finally filtered through a $0.2 \mu \mathrm{m}$ filter to eliminate any excess of undissolved material. The filtered solution was lyophilized until the solid complex was obtained as an orange-red homogeneous powder. The complex was analyzed by ${ }^{1} \mathrm{H}$ NMR to detect the presence of chemical shift changes with respect to the free drug as indicators of fenretinide inclusion into the cyclodextrin hydrophobic cavity ${ }^{50}$. For the analysis the complex was dissolved in $\mathrm{D}_{2} \mathrm{O}$ and the fenretinide salt in DMSO-d6. The ${ }^{1} \mathrm{H}-\mathrm{NMR}$ spectra were recorded by an Inova $600 \mathrm{MHz}$ High Resolution NMR Spectrometer. The amount of fenretinide incorporated into the complex was spectrophotometrically determined in solutions obtained by dissolving known amounts of the solid complex in a water:ethanol $(1: 1, \mathrm{v}-\mathrm{v})$ mixture with the aim to dissociate the drug from the complex and maintain its solubilization in the aqueous phase in the presence of ethanol. The hydro-alcoholic solutions were analyzed for their fenretinide content at the maximum wavelength of the fenretinide absorption $(360 \mathrm{~nm})$. The aqueous solubility of fenretinide complexed with 2hydroxypropyl- $\beta$-cyclodextrin was measured in comparison with the pure drug. Excess amounts of the complex or pure drug were placed in water, stirred $12 \mathrm{~h}$ at $25^{\circ} \mathrm{C}$ in the dark to saturation of the aqueous phase and filtered through $0.2 \mu \mathrm{m}$ filters. After filtration the saturated solutions were spectrophotometrically analysed at $360 \mathrm{~nm}$ for their fenretinide content.

\section{Release studies}

Release studies of fenretinide from the complex were carried out by placing saturated aqueous solutions of the complex in a dialysis membrane bag ( $\mathrm{Mw}$ cutoff $5 \mathrm{KD}$ ), allowing diffusion only to the free drug and not to the complex. The membrane separated the saturated aqueous solution of the complex from a receiving compartment containing an aqueous buffer (PBS pH 7.4) and 1-octanol $(10: 1 \mathrm{v}: \mathrm{v}$ ratio) used to extract the drug diffused through the dialysis membrane and to simulate the presence of hydrophobic absorbing phases such as biomembranes ${ }^{51}$. The release of the drug from the complex was evaluated by a spectrophotometric analysis of the complex solution in the releasing compartment at the maximum absorption wavelength of fenretinide $(360 \mathrm{~nm})$ at appropriate time points.

\section{Pharmacokinetics studies}

The experiments were performed in CD1 female mice, 7 weeks of age, (body weight $25 \pm 2 \mathrm{~g}$ ) obtained from Envigo RMS SrL (Udine, Italy). Mice were maintained under specific-pathogen-free conditions with constant temperature and humidity, according to the institutional guidelines of the Istituto Superiore di Sanità Animal Care Committee. Animal experimentation was conducted in conformance with the following laws, regulations, and policies governing the care and use of laboratory animals: Italian Governing Law (D. 1. 26/2014; Authorization n.19/ 2008-A issued March 6, 2008 by the Ministry of Health). The pharmacokinetics of NanoFEN, administered by oral or intravenous route, was investigated in mice receiving the formulation at doses corresponding to $5 \mathrm{mg} / \mathrm{kg}$ of fenretinide. The intravenous administration was carried out by injection in the tail vein of an intravenous bolus of NanoFEN dissolved in water or PureFEN suspended in a mixture of ethanol $10 \%$ in saline as a comparison. For the oral administration the mice were gavaged the with NanoFEN dissolved in water or with the reconstituted content of the NCI capules, i.e., fenretinide $(100 \mathrm{mg})$ in corn oil $(704 \mathrm{mg})$ and Polysorbate $80(60 \mathrm{mg})$ as a comparison. After the intravenous or oral treatments, a series of blood samples were taken at 15 and $30 \mathrm{~min}$ and at 1, 2, $4,8,10,24$ and $48 \mathrm{~h}$. Blood was collected in heparinized 
tubes from the retro-orbital plexus of the mice under isoflurane anesthesia. To obtain plasma, blood samples were centrifuged at $4000 \mathrm{rpm}$ for $10 \mathrm{~min}$ at $4{ }^{\circ} \mathrm{C}$. The samples were stored at $-20^{\circ} \mathrm{C}$ until analysis. The total concentration of fenretinide in plasma of mice was determined according to a method previously described ${ }^{52}$. Briefly, aliquot of $200 \mu \mathrm{l}$ of each plasma sample was added to $400 \mu \mathrm{l}$ of Acetonitrile $\left(\mathrm{CH}_{3} \mathrm{CN}\right)$ containing $125 \mu \mathrm{g} / \mathrm{mL}$ Butylated hydroxytoluene (BHT), and the mixture was vortexed and centrifuged to pellet the precipitated proteins. The recovered supernatants were analysed on a liquid chromatograph (Perkin-Elmer, Norwall, CT) fitted with a $\mathrm{C} 18(50 \mathrm{~mm} \times 2.0 \mathrm{~mm}, 5 \mu \mathrm{m})$ reverse-phase column and a C18 precolumn (Perkin-Elmer, Milan, Italy). The mobile phase consisted of $\mathrm{CH}_{3} \mathrm{CN}: \mathrm{H} 2 \mathrm{O}: \mathrm{CH}_{3} \mathrm{COOH}$ (75:2:2, v:v:v) delivered at a flow rate of $2 \mathrm{~mL} / \mathrm{min}$. Detection was carried out with a Perkin-Elmer LC95 absorbance detector at $362 \mathrm{~nm}$. N-(4-ethoxyphenyl)-retinamide (EPR) was used as internal standard. For the quantitative evaluation, reference standard curves were set up in control mouse plasma with different known amounts of NanoFEN or PureFEN. The measured fenretinide concentration-vs-time data were elaborated by non-compartmental analysis (WinNonLin software v3) to obtain the main pharmacokinetic parameters of NanoFEN.

\section{Animal procedures}

All animal procedures were performed according to the Italian national animal experimentation guidelines (D. L.116/92) upon approval of the experimental protocol by the Italian Ministry of Health's Animal Experimentation Committee (DM n. 292/2015 PR 23/4/2015). 6-week-old female NOD.Cg-Prkdcscid Il2rgtm1Wjl/SzJ (NSG) mice were purchased from The Jackson Laboratory (Bar Harbor, Maine, USA) for colon and lung cancer xenografts. Tumors were measured twice weekly by an external digital caliper, and volumes were calculated using the following formula: $\pi / 6 \times \mathrm{d} 2 \times \mathrm{D}$, where $\mathrm{d}$ and $\mathrm{D}$ represent shorter and longer tumor measurements, respectively. Drug treatments started when tumor volume was 50$100 \mathrm{~mm}^{3}$ : mice were randomized in control and treatment group and treated with NanoFEN $10 \mathrm{mg} / \mathrm{Kg} /$ threeweekly/ intraperitoneally (IP), chemotherapeutic agent combinations as cisplatin $3 \mathrm{mg} / \mathrm{kg} /$ biweekly $/ \mathrm{IP}+$ pemetrexed $200 \mathrm{mg} / \mathrm{kg} /$ biweekly/IP or cisplatin $3 \mathrm{mg} / \mathrm{kg} /$ biweekly/IP +gemcitabine $60 \mathrm{mg} / \mathrm{kg} /$ biweekly/IP or 5 -fluorouracil $12,5 \mathrm{mg} / \mathrm{Kg}+$ oxaliplatin $5 \mathrm{mg} / \mathrm{Kg} /$ biweekly/IP. Control animals were treated with vehicle only. Tumors were measured at the indicated timepoints and mice were monitored for signs of drug-induced toxicity and weighed regularly. At the end of experiments, tumors were collected and dissociated to obtain cell suspension for subsequent studies or monitored to evaluate tumor growth rate after treatment interruption. Relative tumor growth is indicated as ratio of tumor volume at the indicated days after drug suspension versus volume at the last day of treatment. Animals were euthanized according to the national Animal Welfare Guidelines.

\section{Lentiviral infection}

Primary spheroid cultures of colon and lung cancer cells were stably transduced with mVenus-p27K ${ }^{-28}$ or TOPGFP.mCherry (purchased from Addgene, Cambridge, MA, USA) using ProFection ${ }^{\circledR}$ Mammalian Transfection System from Promega (Madison, Wisconsin, USA) following manufacturer's instructions.

\section{Metabolite extraction}

Samples were extracted following the protocol by D'Alessandro et $\mathrm{al}^{53}$. Samples were resuspended in $0.15 \mathrm{~mL}$ of ice cold ultra-pure water $(18 \mathrm{M} \Omega)$ to lyse cells, then the tubes were plunged into a water bath at $37^{\circ} \mathrm{C}$ for $0.5 \mathrm{~min}$. Samples were mixed with $0.6 \mathrm{~mL}$ of $-20^{\circ} \mathrm{C}$ methanol and then with $0.45 \mathrm{~mL}$ chloroform. Subsequently, $0.15 \mathrm{~mL}$ of ice cold ultra-pure water were added to each tube and then transferred to $-20^{\circ} \mathrm{C}$ for $2-8 \mathrm{~h}$. An equivalent volume of acetonitrile was added to the tube and transferred to $4{ }^{\circ} \mathrm{C}$ for $20 \mathrm{~min}$. Samples were thus centrifuged at $10,000 \times g$ for $10 \mathrm{~min}$ at $4{ }^{\circ} \mathrm{C}$. Finally, samples were dried in a rotational vacuum concentrator (RVC 2-18-Christ Gmbh; Osterode am Harz, Germany) and re-suspended in $200 \mu \mathrm{l}$ of water, $5 \%$ formic acid and transfer to glass auto-sampler vials for LC/MS analysis.

\section{Rapid resolution reversed-phase HPLC}

An Ultimate 3000 Rapid Resolution HPLC system (LC Packings, DIONEX, Sunnyvale, USA) was used to perform metabolite separation. The system featured a binary pump and vacuum degasser, well-plate autosampler with a sixport micro-switching valve, a thermostated column compartment. Samples were loaded onto a Reprosil C18 column $(2.0 \mathrm{~mm} \times 150 \mathrm{~mm}, 2.5 \mu \mathrm{m}$ - Dr Maisch, Germany) for metabolite separation. For lipids multi-step gradient program was used. It started with $8 \%$ solvent $\mathrm{A}$ (ddH20, 20 mmol L-1 ammonium formiate; pH 5) to 6\% solvent A for $3 \mathrm{~min}$ than to $2 \%$ solvent A for $35 \mathrm{~min}$ and finally to $100 \%$ solvent B (methanol) in $30 \mathrm{~min}$. At the end of gradient, the column was reconditioned with $8 \%$ solvent $A$ for $5 \mathrm{~min}$.

\section{Mass spectrometry analysis through microTOF-Q}

Mass spectrometry analysis was carried out on an electrospray hybrid quadrupole time of Flight mass spectrometer MicroTOF-Q (Bruker-Daltonik, Bremen, Germany) equipped with an ESI-ion source. MS analysis was carried out capillary voltage $2800 \mathrm{~V}$, nebulizer $45 \mathrm{psi}$ and dry gas of $91 / \mathrm{min}$, scan mode $100-1500 \mathrm{~m} / \mathrm{z}$. Data 
were acquired with a stored mass range of $100-2000 \mathrm{~m} / z$. Tandem mass spectrometry (MS/MS) is used for lipid species structural characterization. Automatic isolation and fragmentation (AutoMSn mode) was performed on the four most intense ions simultaneously throughout the whole scanning period ( $30 \mathrm{~min}$ per run). Calibration of the mass analyzer is essential in order to maintain an high level of mass accuracy. Instrument calibration was performed externally every day with a sodium formate solution consisting of $10 \mathrm{mM}$ sodium hydroxide in $50 \%$ isopropanol, water, $0.1 \%$ formic acid. Automated internal mass scale calibration was performed through direct automated injection of the calibration solution at the beginning and at the end of each run by a 6-port divertvalve.

\section{Real time PCR}

RNA from vehicle and treated xenografts was extracted with TRIzol (Thermo Fisher Scientific, Waltham, Massachusetts, USA) following manufacturer's instructions. $1 \mu \mathrm{g}$ of RNA were reverse transcribed with M-MLV reverse transcriptase (Thermo Fisher Scientific) and $50 \mathrm{ng}$ of cDNA were used as template in the PCR reactions. Specific probes used for NANOG (Hs04399610_g1), SOX 2 (Hs04260357_g1) and POU5F1 (Hs00999634_gH) were all from Thermo Fisher Scientific. Normalization was performed using $\beta$-actin (Hs00194899_m1) as reference. Values were expressed in terms of $2^{-\Delta \overline{\Delta C t}}$ where $\Delta \Delta \mathrm{Ct}=$ $\Delta \mathrm{Ct}$ sample $-\Delta \mathrm{Ct}$ calibrator. $\Delta \mathrm{Ct}$ is the difference in threshold cycles between the specific RNA target and reference gene amplicons given by StepOne Plus RealTime PCR software by negative correlation with an internal reference dye (ROX).

\section{Reverse-phase protein array}

For RPPA experiments, primary lung cancer spheroid cells (LCSCs) and commercial lung cancer cell lines were left untreated or treated with NanoFEN (IC50 concentration) as follows: LCSC1 $(4.3 \mu \mathrm{M})$, LCSC 2 (1.9 and $3.8 \mu \mathrm{M})$ LCSC $3(50 \mathrm{nM}$ and $1 \mu \mathrm{M})$ LCSC $4(0.9 \mu \mathrm{M})$ LCSC $5(4.2 \mu \mathrm{M})$ LCSC $6(4.9 \mu \mathrm{M}) \mathrm{H} 292(29 \mu \mathrm{M})$. Following $48 \mathrm{~h}$ treatment of the six LCSCs and H292 commercial cell line, cell pellets were promptly lysed in a buffer containing T-PER reagent (Thermo Fisher Scientific), $300 \mathrm{mM} \mathrm{NaCl}$ (J.T.Baker; Avantor Performance Materials, Center Valley, PA), protease and phosphatase inhibitors cocktails (Sigma-Aldrich) and stored at $-80^{\circ} \mathrm{C}$. In order to prepare protein samples for RPPA printing, protein lysates were allowed to thaw on ice, their total protein concentration was measured using the Bradford reagent method (Thermo Fisher Scientific) and the volume corresponding to $50 \mu \mathrm{g}$ of total protein was used to further dilute samples to a final concentration of $0.5 \mathrm{mg} / \mathrm{mL}$, as follows: $50 \% 2 \mathrm{X}$ Tris-Glycine SDS Sample
Buffer (Life Technologies Corporation, Carlsbad, CA), up to $47.5 \%$ sample volume and T-PER reagent (Thermo Fisher Scientific) and 2.5\% Tris (2-carboxyethyl)phosphine hydrochloride (TCEP) reagent (Thermo Fisher Scientific). RPPA lysates were boiled for $3 \mathrm{~min}$ and stored at $-80^{\circ} \mathrm{C}$ until further processing. Prior to printing onto nitrocellulose-coated slides (GRACE Bio-Labs, Bend, OR) using a robotic arrayer (Aushon Biosystems, Billerica, MA, USA), RPPA lysates were allowed to thaw up to room temperature and boiled for an additional $3 \mathrm{~min}$. All samples were printed in technical triplicate as neat and 1:4 dilution pairs. Reference standard lysates, i.e., HeLa + Pervanadate (Becton, Dickinson and Company, Franklin Lakes, NJ, USA), A431+EGF (Becton, Dickinson and Company), Jurkat+Etoposide (Cell Signaling Technology) and Jurkat+Calyculin A (Cell Signaling Technology), were printed in 10-point decreasing mixtures of treated to untreated samples as positive and quality controls of antibody staining. Each reference standard curve was printed in technical triplicates at a final concentration of $0.5 \mathrm{mg} / \mathrm{mL}$. A selected subset of the printed microarray slides were stained with Sypro Ruby Protein Blot Stain (Thermo Fisher Scientific) to estimate sample total protein concentration and the remaining slides were stored under desiccated conditions at $-20^{\circ} \mathrm{C}$. Immediately before antibody staining, printed slides were treated with $1 \mathrm{X}$ Reblot Mild Solution (Chemicon, Burlington, MA, USA) for $15 \mathrm{~min}$, washed $2 \times 5 \mathrm{~min}$ with PBS and incubated for $2 \mathrm{~h}$ in blocking solution containing 2\% I-Block (Applied Biosystems, Foster, CA, USA) and 0.1\% Tween-20 in PBS. Immunostaining was carried out using a tyramide-biotin signal amplification kit (DAKO, Santa Clara, CA, USA). Primary antibody binding was detected using a biotinylated goat anti-rabbit IgG $\mathrm{H}+\mathrm{L}$ (diluted at 1:7500; Vector Laboratories, Burlingame, CA, USA) or rabbit anti-mouse Ig (diluted at 1:10, DAKO) followed by streptavidinconjugated IRDye ${ }^{\oplus}$-680LT fluorophore (LI-COR Biosciences, Lincoln, Nebraska, USA. Primary antibodies undergo pre- and post-RPPA validation for single band specificity by western-blot using complex cellular lysates. Negative control slides, incubated only with secondary antibody were included in each staining run. All Sypro Ruby, i.e., per-spot total protein content,as well as immunostained slides were scanned using a Tecan Power Scanner $^{\text {TwT }}$ (Tecan Group Ltd, Männedorf, Switzerland) at $5 \mu \mathrm{m}$ resolution. Acquired images were analyzed with MicroVigene v5.2, http://www.vigenetech.com/ MicroVigene.htm (VigeneTech Inc, Carlisle, MA, USA), for spot detection, local and negative control background subtraction, replicate averaging and total protein normalization. RPPA data analysis was performed by means of 'R' v3.5.0 https://www.R-project.org/ (R Foundation for Statistical Computing) and 'RStudio' v1.1.414 https:// www.rstudio.com/ (RStudio), using the following installed 
packages:base, plyr, tidyverse, FactoMineR, factoextra, RColorBrewer, Bioconductor and shiny. Custom ' $R$ ' scripts for slide quality control, internal standardization and two-way hierarchical clustering (correlation distance and complete method) are available upon request. A detailed list of antibodies used for RPPA is available in Supplementary Table 1.

\section{Flow cytometry}

For flow cytometry analysis, primary lung and CRC spheroid cultures transduced with the mVenus $\mathrm{p} 27 \mathrm{~K}^{-}$ and treated with NanoFEN, oxaliplatin or gemcitabine were dissociated at a single cell level mechanically or with TrypLE Express (Thermo Fisher Scientific) respectively. Cells were resuspended at a concentration of 500.000 cells $/ \mathrm{mL}$ of PBS $/ 0.4 \%$ BSA/0.5 M EDTA. The percentage of Venus positive cells was analyzed with a FACSCanto flow cytometer (Becton Dickinson) equipped with a DIVA software. $10 \mu \mathrm{g} / \mathrm{mL} \quad 7$-aminoactinomycin D (SigmaAldrich) was used for dead cells exclusion. The same procedure was applied for primary CRC spheroids transduced with the TOP-GFP.mCherry construct treated with NanoFEN, oxaliplatin and 5-fluorouracil for in vitro and ex vivo experiments.

\section{Measurement of intracellular ROS levels}

The intracellular generation of ROS was measured using the oxidation-sensitive fluorescent dye 5chloromethylfluorescein diacetate (CM- $\left.\mathrm{H}_{2} \mathrm{DCFDA}\right)$, with the conversion of $\mathrm{CM}-\mathrm{H}_{2}$ DCFDA to dichlorofluorescein (DCF) assessed as previously described ${ }^{54}$. An equal number of dissociated spheroid cells $\left(2.5 \times 10^{5}\right)$ were seeded in 6-well cell culture plates. Briefly, each cell line was incubated with NanoFEN at IC50 concentration and pre-treated for $1 \mathrm{~h}$ with the antioxidant $\mathrm{N}$-Acetyl-Cysteine (NAC). At different times, the cells were washed twice with pre-warmed Phosphate Saline Buffer (PBS) and then incubated in the same buffer containing $5 \mu \mathrm{M} C \mathrm{CM}$ $\mathrm{H}_{2}$ DCFDA with DMSO alone at $37^{\circ} \mathrm{C}$. The fluorescence intensity of DCF was measured at $527 \mathrm{~nm}$ emission wavelength after excitation at $492 \mathrm{~nm}$ at $1 \mathrm{~h}$ intervals for up to $48 \mathrm{~h}$ using a Flow Cytometer Accuri (Becton Dickinson). An increase in fluorescence intensity as arbitrary units indicated the net generation of intracellular ROS.

\section{Western blotting}

Immunoblotting was performed as previously described $^{55}$. Briefly, whole cell extracts were obtained by incubating the cells in lysis buffer supplemented with protease and phosphatase inhibitor cocktail. Lysates concentration was determined and equal amounts of proteins were loaded on a $4-12 \%$ precast gel (Thermo Fisher Scientific) and transferred to nitrocellulose membranes. Blots were blocked with TBST 5\% non-fat dry milk (Bio-Rad Laboratories, Hercules, CA, USA) and incubated overnight at $4{ }^{\circ} \mathrm{C}$ with primary antibodies (described in the Antibodies and Reagents section) then incubated for 45 min with secondary HRP-conjugated antibodies dissolved in TBST/1\% BSA. Chemiluminescent signals were detected with Amersham ECL prime or select western blotting detection reagent (GE Healthcare Life Sciences, Barrington, IL, USA). Images were taken and analyzed with Bio-Rad ChemiDoc Imagers (Bio-Rad Laboratories).

\section{Viability assay}

Cell viability upon treatment with NanoFEN, zVAD, NAC, 3-MA, Myriocin, PPMP, GT11, SA and Rapamycin was determined by CellTiter-Glo luminescent cell viability assay (Promega, Madison, WI, USA) according to the manufacturer's directions. Briefly, lung and colorectal spheroids were dissociated mechanically or with TrypLE Express (Thermo Fisher Scientific) respectively and seeded $3 \times 10^{3}$ per well in 96-well plates (three replicates per experimental point) in serum-free medium, and incubated in a humidified atmosphere at $37^{\circ} \mathrm{C}, 5 \% \mathrm{CO}_{2}$. Cells were treated with the respective compounds and analysed after the appropriate time period as described in detail in Figure Legends. Luminescence was detected with a DTX880 multimode microplate reader (Beckman Coulter, Brea, CA, USA).

\section{Statistical analysis}

Analyses were performed using GraphPad Prism version 4.0 for Windows (GraphPad Software) with nonpaired double-tailed t-test (after verifying normal distribution of the population with Shapiro-Wilk test) or with one-way ANOVA where appropriate. Results are presented as the mean \pm standard deviation (SD) or standard error of the mean (SEM) where appropriate. Statistical significance is expressed as ${ }^{*} P<0.05$; ${ }^{* *} P<0.01$ and ${ }^{* * *} P<0.001$.

\footnotetext{
Acknowledgements

We thank Paola Di Matteo and Stefano Guida for excellent technical assistance, Antonio Di Virgilio and Daniele Macchia for their essential contribution for in vivo experiments. This work was supported by the following grants: AIRC IG 2017 Ref: 20744, AIRC IG 2014 Ref: 15749, ERA-NET TRANSCAN Ref TACTIC JTC 2014 to A.Z., by Awards 2015 Sapienza University of Rome C26H15ZKWL grant to F.L.T. and by AIRC $5 \times 1000$ Molecular Clinical Oncology Extension Program Ref 9979 grant to R.D.M., G.M. and M.T.
}

\section{Author details}

'Department of Pharmacy and Biotechnology, University of Bologna via San Donato 19/2, 40127 Bologna, Italy. ${ }^{2}$ Department of Oncology and Molecular Medicine, Istituto Superiore di Sanità, Viale Regina Elena 299, 00161 Rome, Italy. ${ }^{3}$ Center for Gender-Specific Medicine, Istituto Superiore di Sanità, Viale Regina Elena 299, 00161 Rome, Italy. ${ }^{4}$ Department of Experimental Medicine and Surgery, University of Rome Tor Vergata, Via Montpellier 1, 00133 Rome, Italy. ${ }^{5}$ RPPA Unit, Proteomics, Core Facilities, Istituto Superiore di Sanità, Viale Regina Elena 299, 00161 Rome, Italy. ${ }^{6}$ Core Facilities, Istituto Superiore di Sanità, Rome, Italy. ${ }^{7}$ Department of Biomedical and Biotechnological Sciences BIOMETEC, University of Catania, via Santa Sofia 97, 95123 Catania, Italy. ${ }^{8}$ DAFNE 
Department, University Tuscia, Via S. Camillo de Lellis, 01100 Viterbo, Italy. ${ }^{9}$ Division of Cellular Therapy, The Institute of Medical Science, The University of Tokyo, Minato-ku, Tokyo 108-8639, Japan. ${ }^{10}$ Division of Stem Cell Signaling, The Institute of Medical Science, The University of Tokyo, 4-6-1 Shirokanedai, Minato-ku, Tokyo 108-8639, Japan. ${ }^{11}$ Center of Animal research and Welfare, Istituto Superiore di Sanità, Rome, Italy. ${ }^{12}$ Environment and Health Department, Istituto Superiore di Sanita', Rome, Italy. ${ }^{13}$ Surgical Sciences and Emergency Department, Division of Emergency \& Trauma Surgery, Emergency Department, Policlinico Umberto I/Sapienza University, Viale del Policlinico 155, 00161 Rome, Italy. ${ }^{14}$ Genetics and Rare Diseases Research Division, Ospedale Pediatrico Bambino Gesù, Viale di San Paolo 15, 00146 Rome, Italy. ${ }^{15}$ Institute of General Pathology, Catholic University of the Sacred Heart, Largo Francesco Vito 1, 00168 Rome, Italy

\section{Author contributions}

I.O. performed the generation and chemical validation of the fenretinidecyclodextrin complex, F.F., L.B., M.L.D.A., A.C., A.E., G.S., P.C., A.P., M.B. performed experiments, K.F., V.S. and M.Sp. performed in vivo experiments, M.Si. performed Reverse Phase Protein microArray, A.Bo., performed FACS analyses, A.Br., M.T., performed genetic analyses of spheroid cultures. L.Z. performed lipidomic analysis, T.O., T.K. generated the mVenus p27K- vector, F.L.T. provided clinical samples, A.G. performed statistical analyses, G.M. provided essential supervision. R.D.M. and A.Z. devised the experiments and supervised the project. A.Z. wrote the manuscript.

\section{Conflict of interest}

The authors declare that they have no conflict of interest.

\section{Publisher's note}

Springer Nature remains neutral with regard to jurisdictional claims in published maps and institutional affiliations.

Supplementary Information accompanies this paper at (https://doi.org/ 10.1038/s41419-019-1775-y).

Received: 17 June 2019 Accepted: 26 June 2019

Published online: 23 July 2019

\section{References}

1. Kreso, A. \& Dick, J. E. Evolution of the cancer stem cell model. Cell Stem Cell 14, 275-291 (2014).

2. Zeuner, A. The secret life of quiescent cancer stem cells. Mol. Cell Oncol. 2 e968067 (2015).

3. Garaventa, A. et al. Phase I trial and pharmacokinetics of fenretinide in children with neuroblastoma. Clin. Cancer Res. 9, 2032-2039 (2003).

4. Maurer, B. J. et al. Phase I trial of fenretinide delivered orally in a novel organized lipid complex in patients with relapsed/refractory neuroblastoma: a report from the New Approaches to Neuroblastoma Therapy (NANT) consortium. Pediatr. Blood Cancer 60, 1801-1808 (2013).

5. Moore, M. M. et al. A phase II study of fenretinide in patients with hormone refractory prostate cancer: a trial of the Cancer Therapeutics Research Group. Cancer Chemother. Pharmacol. 66, 845-850 (2010).

6. Schneider, B. J. et al. Phase II trial of fenretinide (NSC 374551) in patients with recurrent small cell lung cancer. Investig. New Drugs 27, 571-578 (2009).

7. Veronesi, U. et al. Fifteen-year results of a randomized phase III trial of fenretinide to prevent second breast cancer. Ann. Oncol. 17, 1065-1071 (2006).

8. Villablanca, J. G. et al. Phase II study of oral capsular 4hydroxyphenylretinamide (4-HPR/fenretinide) in pediatric patients with refractory or recurrent neuroblastoma: a report from the Children's Oncology Group. Clin. Cancer Res. 17, 6858-6866 (2011).

9. Reynolds, C. P., et al. High plasma levels of fenretinide (4-HPR) were associated with improved outcome in a phase II study of recurrent ovarian cancer: a study by the California Cancer Consortium. J Clin Oncol. 25 (2007).

10. Puduvalli, V. K. et al. Phase II study of fenretinide (NSC 374551) in adults with recurrent malignant gliomas: a North American Brain Tumor Consortium study. J. Clin. Oncol. 22, 4282-4289 (2004).
11. Vaishampayan, $U$. et al. Phase II trial of fenretinide in advanced renal carcinoma. Investig. New Drugs 23, 179-185 (2005).

12. Villablanca, J. G. et al. Phase I trial of oral fenretinide in children with high-risk solid tumors: a report from the Children's Oncology Group (CCG 09709). J. Clin Oncol. 24, 3423-3430 (2006).

13. Jasti, B. R., et al. Phase I clinical trial of fenretinide (NSC374551) in advanced solid tumors. Proc. Am. Soc. Clin. Oncol. 20 (2001).

14. Maurer, B. J. et al. Improved oral delivery of N-(4-hydroxyphenyl)retinamide with a novel LYM-X-SORB organized lipid complex. Clin. Cancer Res. 13, 3079-3086 (2007).

15. Kummar, S. et al. Phase I trial of fenretinide lym-x-sorb oral powder in adults with solid tumors and lymphomas. Anticancer Res. 31, 961-966 (2011).

16. Mohrbacher, A. M. et al. Phase I study of fenretinide delivered intravenously in patients with relapsed or refractory hematologic malignancies: a California Cancer Consortium Trial. Clin. Cancer Res. 23, 4550-4555 (2017).

17. Cooper, J. P., Reynolds, C. P., Cho, H. \& Kang, M. H. Clinical development of fenretinide as an antineoplastic drug: pharmacology perspectives. Exp. Biol. Med 242, 1178-1184 (2017).

18. Brewster, M. E. \& Loftsson, T. Cyclodextrins as pharmaceutical solubilizers. Adv Drug Deliv. Rev. 59, 645-666 (2007).

19. Islam, W. et al. Augmentation of the enhanced permeability and retention effect with nitric oxide-generating agents improves the therapeutic effects of nanomedicines. Mol. cancer Ther. 17, 2643-2653 (2018).

20. Camerini, T. et al. Safety of the synthetic retinoid fenretinide: long-term results from a controlled clinical trial for the prevention of contralateral breast cancer. J. Clin. Oncol. 19, 1664-1670 (2001).

21. Cobleigh, M. A. et al. Phase $\mathrm{I} / \mathrm{I}$ trial of tamoxifen with or without fenretinide, an analog of vitamin A, in women with metastatic breast cancer. J. Clin. Oncol. 11, 474-477 (1993).

22. Dieter, S. M. et al. Distinct types of tumor-initiating cells form human colon cancer tumors and metastases. Cell Stem Cell 9, 357-365 (2011).

23. Francescangeli, F. et al. Proliferation state and polo-like kinase1 dependence of tumorigenic colon cancer cells. Stem Cells 30, 1819-1830 (2012).

24. Vermeulen, L. et al. Single-cell cloning of colon cancer stem cells reveals a multi-lineage differentiation capacity. Proc. Natl. Acad. Sci. USA 105, 13427-13432 (2008)

25. De Angelis, M. L. et al. Cancer stem cell-based models of colorectal cancer reveal molecular determinants of therapy resistance. Stem Cells Transl. Med $\mathbf{5}$, 511-523 (2016).

26. Sette, G. et al. Tyr1068-phosphorylated epidermal growth factor receptor (EGFR) predicts cancer stem cell targeting by erlotinib in preclinical models of wild-type EGFR lung cancer. Cell Death Dis. 6, e1850 (2015).

27. Espina, V., Wulfkuhle, J., Calvert, V. S., Liotta, L. A. \& Petricoin, E. F. 3rd Reverse phase protein microarrays for theranostics and patient-tailored therapy. Methods Mol. Biol. 441, 113-128 (2008).

28. Oki, T. et al. A novel cell-cycle-indicator, mVenus-p27K-, identifies quiescent cells and visualizes G0-G1 transition. Sci. Rep. 4, 4012 (2014).

29. Martins-Neves, S. R., Cleton-Jansen, A. M. \& Gomes, C. M. F. Therapy-induced enrichment of cancer stem-like cells in solid human tumors: Where do we stand? Pharmacol. Res. 137, 193-204 (2018).

30. Vermeulen, L. et al. Wnt activity defines colon cancer stem cells and is regulated by the microenvironment. Nat. cell Biol. 12, 468-476 (2010).

31. Oridate, N. et al. Involvement of reactive oxygen species in $\mathrm{N}$-(4-hydroxyphenyl)retinamide-induced apoptosis in cervical carcinoma cells. J. Natl. Cancer Inst. 89, 1191-1198 (1997).

32. Zhang, $\mathrm{H}$. et al. Preferential eradication of acute myelogenous leukemia stem cells by fenretinide. Proc. Natl. Acad. Sci. USA 110, 5606-5611 (2013).

33. Tanida, I., Ueno, T. \& Kominami, E. LC3 and Autophagy. Methods Mol. Biol. 445, 77-88 (2008)

34. Lovat, P. E. et al. Gangliosides link the acidic sphingomyelinase-mediated induction of ceramide to 12-lipoxygenase-dependent apoptosis of neuroblastoma in response to fenretinide. J. Natl. Cancer Inst. 96, 1288-1299 (2004).

35. Rahmaniyan, M., Curley, R. W. Jr., Obeid, L. M., Hannun, Y. A. \& Kraveka, J. M. Identification of dihydroceramide desaturase as a direct in vitro target for fenretinide. J. Biol. Chem. 286, 24754-24764 (2011).

36. Kraveka, J. M. et al. Involvement of dihydroceramide desaturase in cell cycle progression in human neuroblastoma cells. J. Biol. Chem. 282, 16718-16728 (2007). 
37. Siddique, M. M. et al. Ablation of dihydroceramide desaturase 1, a therapeutic target for the treatment of metabolic diseases, simultaneously stimulates anabolic and catabolic signaling. Mol. Cell. Biol. 33, 2353-2369 (2013).

38. Gagliostro, V. et al. Dihydroceramide delays cell cycle G1/S transition via activation of ER stress and induction of autophagy. Int. J. Biochem. cell Biol. 44 2135-2143 (2012)

39. Lee, Y. S. et al. Myriocin, a serine palmitoyltransferase inhibitor, suppresses tumor growth in a murine melanoma model by inhibiting de novo sphingolipid synthesis. Cancer Biol. Ther. 13, 92-100 (2012).

40. Decensi, A. et al. Randomized double-blind $2 \times 2$ trial of low-dose tamoxifen and fenretinide for breast cancer prevention in high-risk premenopausal women. J. Clin. Oncol. 27, 3749-3756 (2009).

41. Decensi, A. et al. Randomized trial of fenretinide in superficial bladder cancer using DNA flow cytometry as an intermediate end point. Cancer Epidemiol., Biomark. Prev. 9, 1071-1078 (2000).

42. Johansson, $\mathrm{H}$. et al. Effect of fenretinide and low-dose tamoxifen on insulin sensitivity in premenopausal women at high risk for breast cancer. Cancer Res. 68, 9512-9518 (2008)

43. Pienta, K. J., Esper, P. S., Zwas, F., Krzeminski, R. \& Flaherty, L. E. Phase II chemoprevention trial of oral fenretinide in patients at risk for adenocarcinoma of the prostate. Am. J. Clin. Oncol. 20, 36-39 (1997).

44. Aguirre-Ghiso, J. A. \& Sosa, M. S. Emerging topics on disseminated cancer cell dormancy and the paradigm of metastasis. Annu. Rev. Cancer Biol. 2, 377-393 (2018).

45. Adam, A. P. et al. Computational identification of a p38SAPK-regulated transcription factor network required for tumor cell quiescence. Cancer Res. 69 5664-5672 (2009)
46. Bragado, P. et al. TGF-beta2 dictates disseminated tumour cell fate in target organs through TGF-beta-RIII and p38alpha/beta signalling. Nat. cell Biol. 15, 1351-1361 (2013).

47. Kim, R. S. et al. Dormancy signatures and metastasis in estrogen receptor positive and negative breast cancer. PloS One 7, e35569 (2012).

48. De Angelis, M. L. et al. Colorectal cancer spheroid biobanks: multi-level approaches to drug sensitivity studies. Cell Biol. Toxicol. 34, 459-469 (2018).

49. Eramo, A. et al. Identification and expansion of the tumorigenic lung cancer stem cell population. Cell Death Differ. 15, 504-514 (2008).

50. Munoz Botella, S. et al. Analytical applications of retinoid-cyclodextrin inclusion complexes. 1. Characterization of a retinal-beta-cyclodextrin complex. J. Pharm. Biomed. Anal. 14, 909-915 (1996).

51. Esteves, F., Moutinho, C. \& Matos, C. Correlation between octanol/water and liposome/water distribution coefficients and drug absorption of a set of pharmacologically active compounds. J. Liposome Res. 23, 83-93 (2013).

52. Carosio, R. et al. Enhanced anti-neuroblastoma activity of a fenretinide complexed form after intravenous administration. J. Pharm. Pharmacol. 64 228-236 (2012)

53. D'Alessandro, A., D'Amici, G. M., Timperio, A. M., Merendino, N. \& Zolla, L. Docosohaexanoic acid-supplemented PACA44 cell lines and over-activation of Krebs cycle: an integrated proteomic, metabolomic and interactomic overview. J. Proteom. 74, 2138-2158 (2011).

54. Bilski, P., Belanger, A. G. \& Chignell, C. F. Photosensitized oxidation of $2^{\prime}, 7^{\prime}$ dichlorofluorescin: singlet oxygen does not contribute to the formation of fluorescent oxidation product $2^{\prime}, 7^{\prime}$-dichlorofluorescein. Free Radic. Biol. Med. 33, 938-946 (2002).

55. Francescangeli, F. et al. Dynamic regulation of the cancer stem cell compartment by Cripto-1 in colorectal cancer. Cell Death Differ. 22, 1700-1713 (2015). 